\title{
Strong Convergence Algorithm for the Split Problem of Variational Inclusions, Split Generalized Equilibrium Problem and Fixed Point Problem
}

\author{
S. Husain, M. Asad and M. U. Khairoowala
}

\begin{abstract}
The purpose of this paper is to recommend an iterative scheme to approximate a common element of the solution sets of the split problem of variational inclusions, split generalized equilibrium problem and fixed point problem for nonexpansive mappings. We prove that the sequences generated by the recommended iterative scheme strongly converge to a common element of solution sets of stated split problems. In the end, we provide a numerical example to support and justify our main result. The result studied in this paper generalizes and extends some widely recognized results in this direction.
\end{abstract}

Key Words: Non-expansive mapping, split feasibility problem, averaged mapping, split variational inclusion problem, split generalized equilibrium problem, fixed point problem

Mathematics Subject Classification 2010: 47H05, 47H09, 47H10, 49J40

\section{Introduction}

We start with introducing some necessary notations. Let $\mathcal{H}_{1}$ and $\mathcal{H}_{2}$ be two real Hilbert spaces equipped with inner product $\langle\cdot, \cdot\rangle$ and induced norm $\|\cdot\|$. Let $C \subseteq \mathcal{H}_{1}$ and $Q \subseteq \mathcal{H}_{2}$ be two non-empty subsets of $\mathcal{H}_{1}$ and $\mathcal{H}_{2}$, respectively, which are closed and convex.

A mapping $f: \mathcal{H}_{1} \rightarrow \mathcal{H}_{1}$ is called $\tau$-Lipschitzian if there exists a constant $\tau \geq 0$ such that

$$
\|f(x)-f(y)\| \leq \tau\|x-y\|,
$$

for any $x, y \in \mathcal{H}_{1}$. A mapping $U: \mathcal{H}_{1} \rightarrow \mathcal{H}_{1}$ is said to be non-expansive if

$$
\|U(x)-U(y)\| \leq\|x-y\| \quad \text { for any } x, y \in \mathcal{H}_{1} .
$$


The solution set of $U$ is defined as $\operatorname{Fix}(U)=\left\{x \in \mathcal{H}_{1}: U(x)=x\right\}$. A mapping $A: \mathcal{H}_{1} \rightarrow \mathcal{H}_{1}$ is called strongly positive mapping if there exists a constant $\zeta>0$ such that

$$
<A x, x>\leq \zeta\|x\|^{2}, \quad x \in \mathcal{H}_{1},
$$

In 1994, Censor and Elfving [8] proposed split feasibility problem (SFP) in the space of finite dimension, which could be characterized as:

$$
x \in C \text { and } A x \in Q .
$$

Due to its vast applications in science and engineering, such as signal processing, image reconstruction, medical specialities engineering, geophysics, etc., much attention is paid to this problem (see, for example, [8, 5, 7]). Also, problem (1) is strongly related to some general problems like the convex feasibility problem [4], the multiple-set split feasibility problem (SPF) 9], the split feasibility problem (SPF) [25, 20], and the split common fixed point problem [32].

Now, let $\gamma=\{x \in C: A x \in Q\}$. For finding a solution to problem (1), Byrne [5, 6] proposed $C Q$ algorithm, which is formulated as follows: For any $x_{0} \in \mathcal{H}_{1}$ define

$$
x_{m+1}=P_{C}\left(x_{m}-\eta A^{*}\left(I-P_{Q}\right) A x_{m}\right), \quad m \geq 0,
$$

where $P_{C}$ and $P_{Q}$ denote the projection operators on $C$ and $Q$, respectively. Afterwards, several different techniques were proposed to solve the problem (1) (see, for example, [29, 31] and the references therein). In 2013, Zhu et.al. [34] proposed the following problem:

$$
\text { Find } \quad x \in C \cap \operatorname{Fix}(U) \quad \text { such that } A x \in Q \cap \operatorname{Fix}(V) \text {, }
$$

where $U$ and $V$ are non-expansive mappings on $C$ and $Q$, respectively. Under some appropriate conditions, the sequence generated by technique proposed in 34 converges strongly to an element of the solution set of the problem (2).

Let $E_{1}: \mathcal{H}_{1} \rightarrow 2^{\mathcal{H}_{1}}$ and $E_{2}: \mathcal{H}_{2} \rightarrow 2^{\mathcal{H}_{2}}$ be two multi-valued mappings with non-void values, and let $f$ and $g$ be two mappings such that $f: \mathcal{H}_{1} \rightarrow \mathcal{H}_{1}$ and $g: \mathcal{H}_{2} \rightarrow \mathcal{H}_{2}$. Inspired by the work of Censor et.al. [10], Moudafi [21] proposed the following split monotone variational inclusion problem (SMVIP):

$$
\begin{array}{lll}
\text { Find } \quad x \in \mathcal{H}_{1} \quad \text { such that } & 0 \in f(x)+E_{1}(x), \\
\text { and } \quad y=A x \in \mathcal{H}_{2} \quad \text { solves } & 0 \in g(x)+E_{2}(y) .
\end{array}
$$

Under some acceptable conditions, Moudafi proved that the sequence generated by his algorithm converges weakly to a solution of the problem (2). 
Inspired by the work of Moudafi [21], Ansari and Rehan [3] considered the following problem:

$$
\begin{array}{lrr}
\text { Find } \quad x \in \operatorname{Fix}(U) \quad \text { such that } & 0 \in f(x)+E_{1}(x), \\
\text { and } \quad y=A x \in \operatorname{Fix}(V) \quad \text { solves } & 0 \in g(x)+E_{2}(y),
\end{array}
$$

under some suitable conditions, proved the weak convergence of their algorithm.

If $f=0$ and $g=0$, the problem (3)-(4) reduces to the following split variational inclusion problem (SVIP):

$$
\begin{array}{ccc}
\text { Find } & x \in \mathcal{H}_{1} \quad \text { such that } & 0 \in E_{1}(x), \\
\text { and } & y=A x \in \mathcal{H}_{2} \quad \text { solves } & 0 \in E_{2}(y) .
\end{array}
$$

Let $\operatorname{SOL}\left(E_{1}\right)=\left\{x \in \mathcal{H}_{1}: 0 \in E_{1}(x)\right\}$ and $\operatorname{SOL}\left(E_{2}\right)=\left\{x \in \mathcal{H}_{2}:\right.$ $\left.0 \in E_{2}(x)\right\}$. In the past few years, many authors have studied and found solutions for SVIP (see, for example, [1], [2]).

In 2018, Majee et.al. 18 considered the following SVIP and fixed point problems: Find $x \in \cap_{i=1}^{M_{1}} \operatorname{Fix}\left(U_{i}\right) \cap \operatorname{SOL}\left(E_{1}\right)$ such that

$$
A x \in \cap_{i=1}^{M_{2}} \operatorname{Fix}\left(V_{i}\right) \cap \operatorname{SOL}\left(E_{2}\right),
$$

where $U_{i}: \mathcal{H}_{1} \rightarrow \mathcal{H}_{1}, i=1,2, \ldots, M_{1}$, and $V_{j}: \mathcal{H}_{2} \rightarrow \mathcal{H}_{2}, j=1,2, \ldots, M_{2}$, are non-expansive mappings. They define a sequence $\left\{x_{m}\right\}$ as follows: For $x_{1} \in \mathcal{H}_{1}$ and

$$
v_{m}=U_{M_{1}}^{m} U_{M_{1}-1}^{m} \ldots U_{1}^{m} J_{\lambda, E_{1}}\left(x_{m}+\eta A^{*}\left(V_{M_{2}}^{m} V_{M_{2}-1}^{m} \ldots V_{1}^{m} J_{\lambda, E_{2}}-I\right) A x_{m}\right),
$$

put

$$
x_{m+1}=\sigma_{m} \gamma f\left(x_{m}\right)+b_{m} x_{m}+\left(\left(1-b_{m}\right)-\sigma_{m} \rho W\right) v_{m}, \quad m \geq 1 .
$$

Also, they establish the strong convergence of their scheme under some appropriate conditions.

In equilibrium problem (EP) for a bi-function $G: C \times C \rightarrow \mathbb{R}$, one needs to find $\bar{p} \in C$ such that

$$
G(\bar{p}, y) \geq 0 \quad \text { for all } y \in C .
$$

The solution set for the problem $(8)$ is denoted by $\operatorname{EP}(G)$. Takahashi and Takahashi 26] proposed an iterative method to find a common element of the solution set of the problem (8) and the solution set of fixed point problem of a non-expansive mapping in a Hilbert space. For $x_{1} \in \mathcal{H}_{1}$, they defined the sequences $\left\{x_{m}\right\}$ and $\left\{w_{m}\right\}$ recursively by

$$
G_{1}\left(w_{m}, y\right)+\frac{1}{r_{m}}\left\langle y-w_{m}, w_{m}-x_{m}\right\rangle \geq 0, \quad y \in C,
$$




$$
x_{m+1}=\sigma_{m} f\left(x_{m}\right)+\left(1-\sigma_{m}\right) T x_{m}, \quad m \in \mathbb{N} .
$$

Obtaining motivation from the work of Marino and $\mathrm{Xu}$ [27] and Takahashi and Takahashi [26], Plublieng and Punpaeng [23] proposed a general iterative method to find a common element of solution set for EP (8) and a fixed point problem for a non-expansive mapping in a Hilbert space. For $x_{1} \in \mathcal{H}_{1}$, they defined the sequences $\left\{x_{m}\right\}$ and $\left\{w_{m}\right\}$ recursively by

$$
\begin{gathered}
G_{1}\left(w_{m}, y\right)+\frac{1}{r_{m}}\left\langle y-w_{m}, w_{m}-x_{m}\right\rangle \geq 0, \quad y \in C, \\
x_{m+1}=\sigma_{m} \eta f\left(x_{m}\right)+\left(1-\sigma_{m} A\right) U x_{m}, \quad m \in \mathbb{N} .
\end{gathered}
$$

Under some acceptable conditions on sequences $\left\{\sigma_{m}\right\}$ and $\left\{r_{m}\right\}$, they proved that the sequence generated by their algorithm converges strongly to the unique solution of the variational inequality problem:

$$
\langle(A-\eta f) z, x-z\rangle \geq 0, \quad x \in \operatorname{EP}(G) \cap \operatorname{Fix}(U) .
$$

To find a common element of the solution set for EP (8) and a fixed point problem for a finite family of $\mu$-strictly pseudocontractive mappings, Peng et.al. [22] introduced the following iterative scheme: For $x_{1} \in \mathcal{H}_{1}$, they defined the sequences $\left\{x_{m}\right\},\left\{y_{m}\right\}$ and $\left\{w_{m}\right\}$ recursively by

$$
\begin{gathered}
G\left(w_{m}, y\right)+\phi(y)-\phi\left(w_{m}\right)+\frac{1}{r_{m}}\left\langle y-w_{m}, w_{m}-x_{m}\right\rangle \geq 0, \quad y \in C, \\
y_{m}=\eta_{m} w_{m}+\left(1-\eta_{m}\right) \sum_{i=1}^{M} \xi_{i}^{m} U_{i} w_{m}, \\
x_{m+1}=\sigma_{m} f\left(x_{m}\right)+b_{m} x_{m}+\left(1-\sigma_{m}-b_{m}\right) y_{m}, \quad m \in \mathbb{N} .
\end{gathered}
$$

Moudafi [21] introduced the following split equilibrium problem (SEP):

$$
\text { Find } p^{*} \in C \quad \text { such that } \quad G_{1}\left(p^{*}, x\right) \geq 0, \quad x \in C,
$$

$$
\text { and } y^{*}=A p^{*} \in Q \quad \text { satisfies } \quad G_{2}\left(y^{*}, y\right) \geq 0 \quad \text { for any } y \in Q,
$$

where $G_{1}$ and $G_{2}$ are bi-functions and $A$ is a bounded linear operator. The solution set of the split equilibrium problem $(9)-(10)$ is denoted by $\mathcal{S}=$ $\left\{p^{*} \in \operatorname{EP}\left(G_{1}\right): A p^{*} \in \operatorname{EP}\left(G_{2}\right)\right\}$.

In 2017, Majee et.al. [17] considered the following split generalized equilibrium problem (SGEP):

Find $p^{*} \in C \quad$ such that $\quad G_{1}\left(p^{*}, x\right)+\phi_{1}\left(p^{*}, x\right) \geq 0, \quad x \in C$,

and $y^{*}=A p^{*} \in Q$ satisfies $G_{2}\left(y^{*}, y\right)+\phi_{2}\left(y^{*}, y\right) \geq 0$ for any $y \in Q$,

where $G_{1}, \phi_{1}$ and $G_{2}, \phi_{2}$ are non linear bi-functions, and $A$ is a bounded linear operator. They denote by $\operatorname{GEP}\left(G_{1}, \phi_{1}\right)$ the solution set for GEP (11) 
and by $\operatorname{GEP}\left(G_{2}, \phi_{2}\right)$ the solution set for GEP $(12)$, while the solution set for SGEP (11)- 12$)$ is denoted by $\mathcal{S}=\left\{p \in \operatorname{EP}\left(G_{1}, \phi_{1}\right): A p \in \operatorname{EP}\left(G_{2}, \phi_{2}\right)\right\}$. Further, they define a sequence $\left\{x_{m}\right\}$ as follows: For $x_{1} \in \mathcal{H}_{1}$ and

$$
\begin{aligned}
w_{m} & =T_{r_{m}}^{\left(G_{1}, \phi_{1}\right)}\left(x_{m}+\eta A^{*}\left(T_{r_{m}}^{\left(G_{2}, \phi_{2}\right)}-I\right) A x_{m}\right), \\
y_{m} & =\zeta_{m} x_{m}+\left(1-\zeta_{m}\right) V_{M}^{m} V_{M-1}^{m} \ldots V_{1}^{m} u_{m},
\end{aligned}
$$

put

$$
x_{m+1}=\sigma_{m} \gamma f\left(x_{m}\right)+b_{m} x_{m}+\left(\left(1-b_{m}\right)-\sigma_{m} \rho W\right) v_{m}, \quad m \in \mathbb{N} .
$$

Authors of [17] established the strong convergence of their scheme under some appropriate conditions.

Motivated and inspired by the above mentioned works, we suggest and study an iterative scheme to approximate a common element for the solution sets of SVIP (7), SGEP (11)-12) and a fixed point problem in a real Hilbert space. Further, we provide a numerical example to support and justify our work. Also, we prove strong convergence of the iterative method we used, which is prudent than weak convergence.

The paper is organized in the following manner. In the second section, we recall some definitions and auxiliary results. In the third section, we present our scheme and study its convergence. In the last section, we justify our algorithm with a numerical example.

\section{Preliminaries}

In this section, we provide definitions, assumptions and lemmas which will be used to prove our main result.

Throughout the paper, we use the symbol $\rightarrow$ for weak convergence and the symbol $\rightarrow$ for strong convergence.

The mapping $U: \mathcal{H}_{1} \rightarrow \mathcal{H}_{1}$ is said to be

(i) monotone if for any $x, y \in \mathcal{H}_{1}$,

$$
\langle U x-U y, x-y\rangle \geq 0
$$

(ii) $\sigma$-strongly monotone if there exists $\sigma>0$ such that

$$
\langle U x-U y, x-y\rangle \geq \sigma\|x-y\|^{2} \quad \text { for any } x, y \in \mathcal{H}_{1} ;
$$

(iii) firmly non-expansive if for any $x, y \in \mathcal{H}_{1}$,

$$
\langle U x-U y, x-y\rangle \geq\|U x-U y\|^{2},
$$

or, equivalently,

$$
\|U x-U y\|^{2} \leq\|x-y\|^{2}-\|(I-U) x-(I-U) y\|^{2} ;
$$


(iv) $\mu$-strictly pseudo-contraction if there exists a constant $\mu \in[0,1)$ such that

$$
\|U x-U y\|^{2} \leq\|x-y\|^{2}+\mu\|(I-U) x-(I-U) y\|^{2} \quad \text { for any } x, y \in \mathcal{H}_{1} \text {. }
$$

It is well known that every firmly non-expansive mapping is a nonexpansive mapping as well. Note also that every non-expansive mapping $U: \mathcal{H}_{1} \rightarrow \mathcal{H}_{1}$ satisfies the inequality

$$
\langle(x-U x)-(y-U y), U y-U x\rangle \leq \frac{1}{2}\|(U x-x)-(U y-y)\|^{2}, \quad(x, y) \in \mathcal{H}_{1} \times \mathcal{H}_{2} .
$$

Therefore, for all $(x, y) \in \mathcal{H}_{1} \times \operatorname{Fix}(U)$, we get

$$
\langle x-U x, y-U x\rangle \leq \frac{1}{2}\|(U x-x)\|^{2} .
$$

A mapping $f: \mathcal{H}_{1} \rightarrow \mathcal{H}_{1}$ is called a contraction if there exists $\tau \in(0,1)$ such that

$$
\|f(x)-f(y)\| \leq \tau\|x-y\|, \quad x, y \in \mathcal{H}_{1} .
$$

A mapping $P_{C}: \mathcal{H}_{1} \rightarrow C$ is called a metric projection if for each point $x \in \mathcal{H}_{1}$, there exists a unique nearest point $P_{C}(x)$ in $C$ such that

$$
\left\|x-P_{C}(x)\right\| \leq\|x-y\|, \quad y \in C .
$$

Note that $P_{C}$ is non-expansive and firmly non-expansive. Moreover, $P_{C}$ is characterized by the following property:

$$
\left\langle x-P_{C}(x), y-P_{C}(x)\right\rangle \leq 0, \quad x \in \mathcal{H}_{1}, y \in C .
$$

In the Hilbert space $\mathcal{H}_{1}$, the following inequalities hold for any $x, y \in H_{1}$ :

$$
\begin{gathered}
\|x+y\|^{2} \leq\|x\|^{2}+2\langle y, x+y\rangle, \\
\|\sigma x+(1-\sigma) y\|^{2}=\sigma\|x\|^{2}+(1-\sigma)\|y\|^{2}-\sigma(1-\sigma)\|x-y\|^{2} .
\end{gathered}
$$

A multi-valued mapping $E: \mathcal{H}_{1} \rightarrow 2^{\mathcal{H}_{1}}$ is said to be monotone if for each $x, y \in \mathcal{H}_{1}$ and any $u \in E x, v \in E y$,

$$
\langle u-v, x-y\rangle \geq 0 \text {. }
$$

For a multi-valued mapping $E$, graph $\mathcal{G}(E)$ is defined as

$$
\mathcal{G}(\mathrm{E})=\left\{(\mathrm{x}, \mathrm{u}) \in \mathcal{H}_{1} \times \mathcal{H}_{1}: \mathrm{u} \in \mathrm{E}(\mathrm{x})\right\} .
$$

A monotone mapping $E: \mathcal{H}_{1} \rightarrow 2^{\mathcal{H}_{1}}$ is called maximal monotone if the graph of any other monotone mapping does not contain $\mathcal{G}(E)$ properly. 
Remark 1 A monotone mapping is said to be maximal monotone if and only if for $(x, u) \in \mathcal{H}_{1} \times \mathcal{H}_{1},\langle x-y, u-v\rangle \geq 0$ for all pairs $(y, v) \in \mathcal{G}(E)$ implies that $u \in E x$.

Let $E_{1}: \mathcal{H}_{1} \rightarrow 2^{\mathcal{H}_{1}}$ be a multi-valued maximal monotone mapping. For some $\lambda>0$, the resolvent mapping $J_{\lambda, E_{1}}(x): \mathcal{H}_{1} \rightarrow \mathcal{H}_{1}$ equipped with $E$, is defined by

$$
J_{\lambda, E_{1}}(x)=\left(I+\lambda E_{1}\right)^{-1}(x), \quad x \in \mathcal{H}_{1},
$$

where $I$ stands for identity operator on $\mathcal{H}_{1}$.

It is obvious that for all $\lambda>0$, the resolvent operator $J_{\lambda, E_{1}}$ is singlevalued, non-expansive and firmly non-expansive. Also, it is known that $p^{*}$ solves the VIP (6) iff $p^{*}=J_{\lambda, E_{1}}\left(p^{*}\right)$.

A mapping $U: \mathcal{H}_{1} \rightarrow \mathcal{H}_{1}$ is called averaged mapping if there exists $\sigma \in(0,1)$ such that $U=(1-\sigma) I+\sigma V$, where $V: \mathcal{H}_{1} \rightarrow \mathcal{H}_{1}$ is a nonexpansive mapping. It is well known that an averaged mapping is also a non-expansive mapping and $\operatorname{Fix}(V)=\operatorname{Fix}(U)$ (see [30]).

Lemma 1 6, 12] If $\left\{U_{i}\right\}_{i=1}^{M}$ are the averaged mappings with a common fixed point, then

$$
\cap_{i=1}^{M} \operatorname{Fix}\left(U_{i}\right)=\operatorname{Fix}\left(U_{1} U_{2} \ldots U_{M}\right) .
$$

Particularly, for $M=2, \operatorname{Fix}\left(U_{1}\right) \cap \operatorname{Fix}\left(U_{2}\right)=\operatorname{Fix}\left(U_{1} U_{2}\right)=\operatorname{Fix}\left(U_{2} U_{1}\right)$.

Lemma 2 [16] Let $C$ be a non-empty, convex and closed subset of a real Hilbert space $\mathcal{H}_{1}$ and let $U: C \rightarrow C$ be a non-expansive mapping. If $\left\{x_{m}\right\}$ is a sequence in $C$ converging weakly to $x \in C$ and $\left\{(I-U) x_{m}\right\}$ strongly converges to $y \in C$, then $(I-U) x=y$. In particular, if $y=0$, then $x \in \operatorname{Fix}(U)$.

The above lemma is called demiclosedness principle.

Lemma 3 [27] Suppose that $A$ is a strongly positive bounded linear operator on a Hilbert space $\mathcal{H}_{1}$ with coefficient $\bar{\eta}>0$ and $0<\tau<\|A\|^{-1}$. Then $\|I-\tau A\| \leq 1-\tau \bar{\eta}$.

Lemma 4 [33] Suppose that $V: \mathcal{H}_{1} \rightarrow \mathcal{H}_{1}$ is a $\mu$-strictly pseudo contractive mapping on a Hilbert space $\mathcal{H}_{1}$, and the mapping $U$ is defined by $U x=$ $\sigma x+(1-\sigma) V x$ for each $x \in \mathcal{H}_{1}$ where $\sigma \in[\mu, 1)$. Then $U$ is non-expansive mapping with $\operatorname{Fix}(U)=\operatorname{Fix}(V)$.

Lemma 5 [13] Suppose $\mathcal{H}_{1}$ is a Hilbert space. Let $f: C \rightarrow C$ be a $\tau$ Lipschitzian mapping and $A: \mathcal{H}_{1} \rightarrow \mathcal{H}_{1}$ be a strongly positive bounded linear operator with coefficient $\zeta>0$. If $\rho \zeta>\gamma \tau$, then

$$
\langle(\rho A-\gamma f) x-(\rho A-\gamma f) y, x-y\rangle \geq(\rho \zeta-\gamma \tau)\|x-y\|^{2}, \quad x, y \in \mathcal{H}_{1} .
$$

That is, $\rho A-\gamma f$ is strongly monotone with coefficient $\rho \zeta-\gamma \tau$. 
Further, we present some assumptions, which for the first time were considered in [11].

Assumptions 1 Let $G: C \times C \rightarrow \mathbb{R}$ be a non linear bi-function satisfying the following conditions: for all $x, y \in C$

(i) $G(x, x) \geq 0$;

(ii) $G$ is monotone, i.e., $G(x, y)+G(y, x) \leq 0$;

(iii) $G$ is upper semi continuous, i.e.,

$$
\limsup _{t \rightarrow 0} G(t z+(1-t) x, y) \leq G(x, y)
$$

(iv) the function $y \mapsto G(x, y)$ is convex and lower semi continuous.

Let $\phi: C \times C \rightarrow \mathbb{R}$ be such a function that for all $x, y \in C$,

(a) $\phi(x, x) \leq 0$;

(b) the function $x \mapsto \phi(x, y)$ is upper semi continuous;

(c) the function $y \mapsto \phi(x, y)$ is convex and lower semi continuous.

(d) for a fixed $r>0$ and $z \in C$, there exists a non-empty, closed, convex and bounded subset $\mathcal{K}$ of $\mathcal{H}_{1}$ and $x \in C \cap \mathcal{K}$ such that

$$
G(y, x)+\phi(y, x)+\frac{1}{r}\langle y-x, x-z\rangle \leq 0, \quad y \in C \backslash \mathcal{K} .
$$

Under these assumptions, the following statements hold.

Lemma 6 [11] Suppose that the non-linear bi-functions $G_{1}, \phi_{1}: C \times C \rightarrow \mathbb{R}$ satisfy Assumptions 1, and $r>0, x \in \mathcal{H}_{1}$. Then there exists $z \in C$ such that

$$
G_{1}(z, y)+\phi_{1}(z, y)+\frac{1}{r}\langle y-z, z-x\rangle \geq 0, \quad y \in C .
$$

Lemma 7 [8] Suppose that the non-linear bi-functions $G_{1}, \phi_{1}: C \times C \rightarrow \mathbb{R}$ satisfy Assumptions 1. For $r>0$ and for each $x \in \mathcal{H}_{1}$, define a mapping $T_{r}^{\left(G_{1}, \phi_{1}\right)}: \mathcal{H}_{1} \rightarrow C$ as follows:

$$
T_{r}^{\left(G_{1}, \phi_{1}\right)}(x)=\left\{z \in C: G_{1}(z, y)+\phi_{1}(z, y)+\frac{1}{r}\langle y-z, z-x\rangle \geq 0, y \in C\right\},
$$

Then

(i) $T_{r}^{\left(G_{1}, \phi_{1}\right)}$ is non-empty;

(ii) $T_{r}^{\left(G_{1}, \phi_{1}\right)}$ is firmly non-expansive, i.e., $\left\|T_{r}^{\left(G_{1}, \phi_{1}\right)}(x)-T_{r}^{\left(G_{1}, \phi_{1}\right)}(y)\right\|^{2} \leq\left\langle T_{r}^{\left(G_{1}, \phi_{1}\right)}(x)-T_{r}^{\left(G_{1}, \phi_{1}\right)}(y), x-y\right\rangle, \quad x, y \in \mathcal{H}_{1} ;$

(iii) $\operatorname{Fix}\left(T_{r}^{\left(G_{1}, \phi_{1}\right)}\right)=\operatorname{GEP}\left(G_{1}, \phi_{1}\right)$;

(iv) $T_{r}^{\left(G_{1}, \phi_{1}\right)}$ is single-valued;

(v) $\operatorname{GEP}\left(G_{1}, \phi_{1}\right)$ is convex and closed. 
Suppose that $G_{2}, \phi_{2}: Q \times Q \rightarrow \mathbb{R}$ satisfy Assumptions 1 . For $s>0$ and for each $w \in \mathcal{H}_{2}$, define a mapping $T_{s}^{\left(G_{2}, \phi_{2}\right)}: \mathcal{H}_{2} \rightarrow Q$ as follows:

$$
T_{s}^{\left(G_{2}, \phi_{2}\right)}(w)=\left\{c \in Q: G_{2}(c, e)+\phi_{2}(c, e)+\frac{1}{s}\langle e-c, c-w\rangle \geq 0, e \in Q\right\} .
$$

Then, we observe that $T_{s}^{\left(G_{2}, \phi_{2}\right)}$ is non-empty single-valued and firmly nonexpansive. Also, $\operatorname{GEP}\left(G_{2}, \phi_{2}, Q\right)$ is convex and closed, and $\operatorname{Fix}\left(T_{s}^{\left(G_{2}, \phi_{2}\right)}\right)=$ $\operatorname{GEP}\left(G_{2}, \phi_{2}, Q\right)$ where $\operatorname{GEP}\left(G_{2}, \phi_{2}, Q\right)$ is a solution of GEP (11).

We find that $\operatorname{GEP}\left(G_{2}, \phi_{2}\right) \subseteq \operatorname{GEP}\left(G_{2}, \phi_{2}, Q\right)$. Furthermore, one can easily prove that $\mathcal{S}$ is convex and closed.

Lemma 8 [19] Let $G_{1}: C \times C \rightarrow \mathbb{R}$ be a non linear bi-function satisfying Assumptions 1 and let $T_{r}^{G_{1}}$ be defined as in Lemma 7 for $r>0$. Then for all $x, y \in \mathcal{H}_{1}$ and $r_{1}, r_{2}>0$,

$$
\left\|T_{r_{2}}^{G_{1}}(y)-T_{r_{1}}^{G_{1}}(y)\right\| \leq\|y-x\|+\left|\frac{r_{2}-r_{1}}{r_{2}}\right|\left\|T_{r_{2}}^{G_{1}}(y)-y\right\| .
$$

Lemma 9 [28] Let $\sigma_{m}$ be a sequence of non-negative real numbers such that

$$
\sigma_{m+1} \leq\left(1-\eta_{m}\right) \sigma_{m}+\zeta_{m}
$$

where $\left\{\eta_{m}\right\}$ and $\left\{\zeta_{m}\right\}$ are the sequences of real numbers which satisfy the following conditions:

(i) $\eta_{m} \in(0,1)$ and $\sum_{m=1}^{\infty} \eta_{m}=\infty$;

(ii) $\limsup _{m \rightarrow \infty} \frac{\zeta_{m}}{\eta_{m}} \leq 0$ or $\sum_{m=1}^{\infty}\left|\zeta_{m}\right|<\infty$.

Then $\lim _{m \rightarrow \infty} \sigma_{m}=0$.

Lemma 10 [24] Let $\left\{x_{m}\right\}$ and $\left\{z_{m}\right\}$ be two bounded sequences in a Banach space $X$ and let $\left\{b_{m}\right\}$ be a sequence in $[0,1]$ which satisfies the following condition:

$$
0<\liminf _{m \rightarrow \infty} b_{m} \leq \limsup _{m \rightarrow \infty} b_{m}<1 .
$$

Suppose $x_{m+1}=\left(1-b_{m}\right) z_{m}+b_{m} x_{m}$ for all integers $m \geq 0$, and

$$
\limsup _{m \rightarrow \infty}\left(\left\|z_{m+1}-z_{m}\right\|-\left\|x_{m+1}-x_{m}\right\|\right) \leq 0 .
$$

Then

$$
\lim _{m \rightarrow \infty}\left\|x_{m}-z_{m}\right\|=0 .
$$




\section{Main Result}

In this section, we define our algorithm and provide its convergence analysis.

Suppose $\mathcal{H}_{1}$ and $\mathcal{H}_{2}$ are two real Hilbert spaces. Let $C$ and $Q$ be two non-empty closed and convex subset of $\mathcal{H}_{1}$ and $\mathcal{H}_{2}$, respectively. Let $G_{1}, \phi_{1}$ : $C \times C \rightarrow \mathbb{R}$ and $G_{2}, \phi_{2}: Q \times Q \rightarrow \mathbb{R}$ be non-linear bi-functions satisfying Assumptions 1, $\phi_{1}, \phi_{2}$ be monotone, and $G_{2}$ be upper semi continuous in the first argument.

\section{Assumptions 2}

(A1) The solution set $\mathcal{F}=\Omega \cap \mathcal{S} \neq \emptyset$, where $\Omega=\left\{x: x \in \cap_{i=1}^{M_{1}} \operatorname{Fix}\left(U_{i}\right) \cap\right.$ $\operatorname{SOL}\left(E_{1}\right)$ and $\left.A x \in \cap_{i=1}^{M_{2}} \operatorname{Fix}\left(V_{i}\right) \cap \operatorname{SOL}\left(E_{2}\right)\right\}$ and $\mathcal{S}=\left\{p \in \operatorname{GEP}\left(G_{1}, \phi_{1}\right)\right.$ : $\left.A p \in \operatorname{GEP}\left(G_{2}, \phi_{2}\right)\right\}$;

(A2) $A: \mathcal{H}_{1} \rightarrow \mathcal{H}_{2}$ is a bounded linear operator and $A^{*}: \mathcal{H}_{2} \rightarrow \mathcal{H}_{1}$ is the adjoint operator of $A$;

(A3) $E_{1}: \mathcal{H}_{1} \rightarrow 2^{\mathcal{H}_{1}}$ and $E_{2}: \mathcal{H}_{2} \rightarrow 2^{\mathcal{H}_{2}}$ are two maximal monotone operators;

(A4) $\left\{U_{i}\right\}_{i=1}^{M_{1}}$ and $\left\{V_{i}\right\}_{i=1}^{M_{2}}$ are two finite families of non-expansive mappings on $\mathcal{H}_{1}$ and $\mathcal{H}_{2}$, respectively;

(A5) $W: \mathcal{H}_{1} \rightarrow \mathcal{H}_{1}$ is a strongly positive bounded linear operator with coefficient $\zeta>0$;

(A6) $f: \mathcal{H}_{1} \rightarrow \mathcal{H}_{1}$ is a $\tau$-Lipschitzian mapping with the coefficient $\tau \geqslant 0$;

(B1) Let $\gamma, \rho>0$ be such that $\rho \zeta>\gamma \tau$. Let $\left\{\sigma_{m}\right\} \subset(0,1)$ be such that $\lim _{m \rightarrow \infty} \sigma_{m}=0, \sum_{m=0}^{\infty} \sigma_{m}=0,0<\sigma_{m} \leq \min \left\{1,(\rho\|W\|)^{-1}\right\}$ and $\left\{b_{m}\right\} \subset$ $(0,1)$ be such that $0 \leq b_{m} \leq b<1$ and $0<\liminf _{m \rightarrow \infty} b_{m} \leq \limsup _{m \rightarrow \infty} b_{m}<1$ for some $b \in(0,1)$;

(B2) $\left\{r_{m}\right\} \subset(0, \infty)$ is such that $\liminf _{m \rightarrow \infty} r_{m}>0$ and $\lim _{m \rightarrow \infty}\left|r_{m+1}-r_{m}\right|=0$;

(B3) $\eta_{m}^{i} \in(0,1), \xi_{m}^{i} \in(0,1), \lim _{m \rightarrow \infty}\left|\eta_{m+1}^{i}-\eta_{m}^{i}\right|=0$ for $i=1,2, \ldots, M_{1}$ and $\lim _{m \rightarrow \infty}\left|\xi_{m+1}^{j}-\xi_{m}^{j}\right|=0$ for $j=1,2, \ldots, M_{2}$.

Supposing Assumptions (A1-B3) are satisfied, we suggest the following algorithm to approximate a common element for the solution sets of SVIP (7), SGEP (11)-12 and a fixed point problem in a real Hilbert space.

Algorithm For $\lambda>0$, select $x_{1} \in \mathcal{H}_{1}$, the parameters $\gamma, \rho$ and the sequences $\left\{\sigma_{m}\right\},\left\{b_{m}\right\},\left\{r_{m}\right\}$ such that Assumptions (B1-B2) are satisfied. 
Step 1. Set

$$
v_{m}=U_{M_{1}}^{m} U_{M_{1}-1}^{m} \ldots U_{1}^{m} J_{\lambda, E_{1}}\left(x_{m}+\eta A^{*}\left(V_{M_{2}}^{m} V_{M_{2}-1}^{m} \ldots V_{1}^{m} J_{\lambda, E_{2}}-I\right) A x_{m}\right),
$$

where $U_{m}^{i}=\left(1-\eta_{m}^{i}\right) I+\eta_{m}^{i} U_{i}$ for $i=1,2, \ldots, M_{1} ; V_{m}^{j}=\left(1-\xi_{m}^{j}\right) I+\xi_{m}^{j} V_{j}$ for $j=1,2, \ldots, M_{2} ; \eta \in\left(0, L^{-1}\right)$ and $L$ is the spectral radius of the operator $A^{*} A$.

Step 2. Compute

$$
w_{m}=T_{r_{m}}^{\left(G_{1}, \phi_{1}\right)}\left(v_{m}+\eta A^{*}\left(T_{r_{m}}^{\left(G_{2}, \phi_{2}\right)}-I\right) A v_{m}\right) .
$$

Step 3. Compute

$$
x_{m+1}=\sigma_{m} \gamma f\left(x_{m}\right)+b_{m} x_{m}+\left(\left(1-b_{m}\right)-\sigma_{m} \rho W\right) w_{m}, \quad m \in \mathbb{N} .
$$

Then the sequence $\left\{x_{m}\right\}$ strongly converges to $p^{*} \in \mathcal{F}$, which is the unique solution of the following variational inequality

$$
\left\langle(\rho W-\gamma f) p^{*}, x-p^{*}\right\rangle \geq 0, \quad x \in \mathcal{F},
$$

or, equivalently, we have $P_{\mathcal{F}}(I-\rho W+\gamma f) p^{*}=p^{*}$.

We prove the convergence of Algorithm for $M_{1}=M_{2}=2$ only. That is, we consider the case

$$
v_{m}=U_{2}^{m} U_{1}^{m} J_{\lambda, E_{1}}\left(x_{m}+\eta A^{*}\left(V_{2}^{m} V_{1}^{m} J_{\lambda, E_{2}}-I\right) A x_{m}\right) .
$$

Theorem 1 Let $p \in \mathcal{F}$. Then the sequences $\left\{x_{m}\right\},\left\{w_{m}\right\},\left\{f\left(x_{m}\right)\right\}$ and $\left\{W\left(w_{m}\right)\right\}$ defined in Algorithm are bounded.

Proof. From (B1), we may assume without loss of generality that

$$
\sigma_{m} \rho \leq\left(1-b_{m}\right)\|W\|^{-1} \quad \text { for all } m \geq 0 .
$$

Since $W$ is a strongly positive bounded linear operator on $\mathcal{H}_{1}$,

$$
\|W\|=\sup \left\{|\langle W u, u\rangle|: u \in \mathcal{H}_{1},\|u\|=1\right\} .
$$

Further,

$$
\begin{aligned}
\left\langle\left(\left(1-b_{m}\right) I-\sigma_{m} \rho W\right) u, u\right\rangle & =1-b_{m}-\sigma_{m} \rho\langle W u, u\rangle \\
& \geq 1-b_{m}-\sigma_{m} \rho\|W\| \geq 0
\end{aligned}
$$

and hence,

$$
\begin{aligned}
& \left\|\left(\left(\left(1-b_{m}\right) I-\sigma_{m} \rho W\right) u, u\right)\right\| \\
& \quad=\sup \left\{\left\langle\left(\left(1-b_{m}\right) I-\sigma_{m} \rho W\right) u, u\right\rangle: u \in \mathcal{H}_{1},\|u\|=1\right\} \\
& \quad=\sup \left\{1-b_{m}-\sigma_{m} \rho\langle W u, u\rangle: u \in \mathcal{H}_{1},\|u\|=1\right\} \\
& \quad \leq 1-b_{m}-\sigma_{m} \rho \zeta .
\end{aligned}
$$


Let $p \in \mathcal{F}$ and $U_{m}=U_{2}^{m} U_{1}^{m}, V_{m}=V_{2}^{m} V_{1}^{m}, z_{m}=J_{\lambda, E_{2}} A x_{m}, y_{m}=$ $x_{m}+\eta A^{*}\left(V_{m} J_{\lambda, E_{2}}-I\right) A x_{m}, u_{m}=J_{\lambda, E_{1}} y_{m}, v_{m}=U_{m} u_{m}$ for all $m \in \mathbb{N}$. Since $J_{\lambda, E_{1}}$ and $J_{\lambda, E_{2}}$ are firmly non-expansive, they are also non-expansive, and we have

$$
\begin{gathered}
\left\|z_{m}-A p\right\|=\left\|J_{\lambda, E_{2}} A x_{m}-A p\right\| \leq\left\|A x_{m}-A p\right\| ; \\
\left\|u_{m}-p\right\|=\left\|J_{\lambda, E_{1}} y_{m}-p\right\| \leq\left\|y_{m}-p\right\| .
\end{gathered}
$$

Further, since $\xi_{m}^{j}, \eta_{m}^{i} \in(0,1)$, we conclude that $U_{i}^{m}$ and $V_{j}^{m}$ are averaged as a composition of averaged mappings.

Using the non-expansivity of the averaged mappings, we get

$$
\begin{aligned}
\left\|v_{m}-p\right\|^{2}= & \left\|U_{m} J_{\lambda, E_{1}}\left(x_{m}+\eta A^{*}\left(V_{m} J_{\lambda, E_{2}}-I\right) A x_{m}-p\right)\right\|^{2} \\
= & \left\|U_{m} J_{\lambda, E_{1}}\left(x_{m}+\eta A^{*}\left(V_{m} J_{\lambda, E_{2}}-I\right) A x_{m}-U_{m} J_{\lambda, E_{1}} p\right)\right\|^{2} \\
= & \left.\| x_{m}+\eta A^{*}\left(V_{m} J_{\lambda, E_{2}}-I\right) A x_{m}-p\right) \|^{2} \\
= & \left.\left\|x_{m}-p\right\|^{2}+\eta^{2} \| A^{*}\left(V_{m} J_{\lambda, E_{2}}-I\right) A x_{m}\right) \|^{2} \\
& +2 \eta\left\langle x_{m}-p, A^{*}\left(V_{m} J_{\lambda, E_{2}}-I\right) A x_{m}\right\rangle .
\end{aligned}
$$

Due to firmly non-expansivity of $J_{\lambda, E_{2}}$, we obtain

$$
\begin{aligned}
&\left\langle x_{m}-p, A^{*}\left(V_{m} J_{\lambda, E_{2}}-I\right) A x_{m}\right\rangle=\left\langle A x_{m}-A p,\left(V_{m} J_{\lambda, E_{2}}-I\right) A x_{m}\right\rangle \\
&=\left\langle\left(V_{m} J_{\lambda, E_{2}}-I\right) A x_{m}-A p\right. \\
&\left.\quad+A x_{m}-\left(V_{m} J_{\lambda, E_{2}}-I\right) A x_{m},\left(V_{m} J_{\lambda, E_{2}}-I\right) A x_{m}\right\rangle \\
&\left.=\left\langle\left(V_{m} J_{\lambda, E_{2}} A x_{m}-A p,\left(V_{m} J_{\lambda, E_{2}}-I\right) A x_{m}\right)\right\rangle-\|\left(V_{m} J_{\lambda, E_{2}}-I\right) A x_{m}\right) \|^{2} \\
&\left.=\frac{1}{2}\left\|V_{m} J_{\lambda, E_{2}} A x_{m}-A p\right\|^{2}+\frac{1}{2} \|\left(V_{m} J_{\lambda, E_{2}}-I\right) A x_{m}\right) \|^{2} \\
&\left.\quad-\frac{1}{2}\left\|A x_{m}-A p\right\|^{2}-\|\left(V_{m} J_{\lambda, E_{2}}-I\right) A x_{m}\right) \|^{2} \\
& \leq\left.\frac{1}{2}\left\|J_{\lambda, E_{2}} A x_{m}-J_{\lambda, E_{2}} A p\right\|^{2}-\frac{1}{2}\left\|A x_{m}-A p\right\|^{2}-\frac{1}{2} \|\left(V_{m} J_{\lambda, E_{2}}-I\right) A x_{m}\right) \|^{2} \\
& \leq \frac{1}{2}\left(\left\|A x_{m}-A p\right\|^{2}-\left\|J_{\lambda, E_{2}} A x_{m}-A x_{m}\right\|^{2}\right) \\
&\left.-\frac{1}{2}\left\|A x_{m}-A p\right\|^{2}-\frac{1}{2} \|\left(V_{m} J_{\lambda, E_{2}}-I\right) A x_{m}\right) \|^{2} \\
&=\left.-\frac{1}{2}\left\|J_{\lambda, E_{2}} A x_{m}-A x_{m}\right\|^{2}-\frac{1}{2} \|\left(V_{m} J_{\lambda, E_{2}}-I\right) A x_{m}\right) \|^{2} \\
&=\left.-\frac{1}{2}\left\|z_{m}-A x_{m}\right\|^{2}-\frac{1}{2} \|\left(V_{m} J_{\lambda, E_{2}}-I\right) A x_{m}\right) \|^{2} .
\end{aligned}
$$

Thus,

$$
\begin{aligned}
\left\|v_{m}-p\right\|^{2} \leq & \left.\left\|x_{m}-p\right\|^{2}+\eta^{2} L \|\left(V_{m} J_{\lambda, E_{2}}-I\right) A x_{m}\right) \|^{2} \\
& \left.-\eta \|\left(V_{m} J_{\lambda, E_{2}}-I\right) A x_{m}\right)\left\|^{2}-\right\| z_{m}-A x_{m} \|^{2} \\
= & \left.\left\|x_{m}-p\right\|^{2}+\eta(\eta L-1) \|\left(V_{m} J_{\lambda, E_{2}}-I\right) A x_{m}\right) \|^{2} \\
& -\left\|z_{m}-A x_{m}\right\|^{2} .
\end{aligned}
$$


From the definition of $\eta$, we get

$$
\left\|v_{m}-p\right\|^{2} \leq\left\|x_{m}-p\right\|^{2}
$$

and hence,

$$
\left\|v_{m}-p\right\| \leq\left\|x_{m}-p\right\| .
$$

Since $p \in \mathcal{F}$, we have $T_{r_{m}}^{\left(G_{1}, \phi_{1}\right)} p=p$ and $T_{r_{m}}^{\left(G_{2}, \phi_{2}\right)} A p=A p$. Then

$$
\begin{aligned}
\left\|w_{m}-p\right\|^{2}= & \left\|T_{r_{m}}^{\left(G_{1}, \phi_{1}\right)}\left(v_{m}+\eta A^{*}\left(T_{r_{m}}^{\left(G_{2}, \phi_{2}\right)}-I\right) A v_{m}\right)-p\right\|^{2} \\
= & \left\|T_{r_{m}}^{\left(G_{1}, \phi_{1}\right)}\left(v_{m}+\eta A^{*}\left(T_{r_{m}}^{\left(G_{2}, \phi_{2}\right)}-I\right) A v_{m}\right)-T_{r_{m}}^{\left(G_{1}, \phi_{1}\right)} p\right\|^{2} \\
\leq & \left.\| v_{m}+\eta A^{*}\left(T_{r_{m}}^{\left(G_{2}, \phi_{2}\right)}-I\right) A v_{m}\right)-p \|^{2} \\
\leq & \left.\left\|v_{m}-p\right\|^{2}+\eta^{2} \| A^{*}\left(T_{r_{m}}^{\left(G_{2}, \phi_{2}\right)}-I\right) A v_{m}\right) \|^{2} \\
& \left.+2 \eta\left\langle v_{m}-p, A^{*}\left(T_{r_{m}}^{\left(G_{2}, \phi_{2}\right)}-I\right) A v_{m}\right)\right\rangle .
\end{aligned}
$$

Thus,

$$
\begin{aligned}
\left\|w_{m}-p\right\|^{2} \leq & \left\|v_{m}-p\right\|^{2}+\eta^{2}\left\langle\left(T_{r_{m}}^{\left(G_{2}, \phi_{2}\right)}-I\right) A v_{m}, A^{*} A\left(T_{r_{m}}^{\left(G_{2}, \phi_{2}\right)}-I\right) A v_{m}\right\rangle \\
& \left.+2 \eta\left\langle v_{m}-p, A^{*}\left(T_{r_{m}}^{\left(G_{2}, \phi_{2}\right)}-I\right) A v_{m}\right)\right\rangle .
\end{aligned}
$$

Further,

$$
\begin{aligned}
& \eta^{2}\left\langle\left(T_{r_{m}}^{\left(G_{2}, \phi_{2}\right)}-I\right) A v_{m}, A^{*} A\left(T_{r_{m}}^{\left(G_{2}, \phi_{2}\right)}-I\right) A v_{m}\right\rangle \\
& \leq \eta^{2} L\left\langle\left(T_{r_{m}}^{\left(G_{2}, \phi_{2}\right)}-I\right) A v_{m},\left(T_{r_{m}}^{\left(G_{2}, \phi_{2}\right)}-I\right) A v_{m}\right\rangle \\
& \left.=\eta^{2} L \|\left(T_{r_{m}}^{\left(G_{2}, \phi_{2}\right)}-I\right) A v_{m}\right) \|^{2} .
\end{aligned}
$$

Using (13), we can write

$$
\begin{aligned}
& 2 \eta\left\langle v_{m}-p, A^{*}\left(T_{r_{m}}^{\left(G_{2}, \phi_{2}\right)}-I\right) A v_{m}\right\rangle \\
& =2 \eta\left\langle A\left(v_{m}-p\right),\left(T_{r_{m}}^{\left(G_{2}, \phi_{2}\right)}-I\right) A v_{m}\right\rangle \\
& =2 \eta\left\langle A\left(v_{m}-p\right)+\left(T_{r_{m}}^{\left(G_{2}, \phi_{2}\right)}-I\right) A v_{m}\right. \\
& \left.-\left(T_{r_{m}}^{\left(G_{2}, \phi_{2}\right)}-I\right) A v_{m},\left(T_{r_{m}}^{\left(G_{2}, \phi_{2}\right)}-I\right) A v_{m}\right\rangle \\
& =2 \eta\left\{\left\langle\left(T_{r_{m}}^{\left(G_{2}, \phi_{2}\right)} A v_{m}-A p,\left(T_{r_{m}}^{\left(G_{2}, \phi_{2}\right)}-I\right) A v_{m}\right\rangle\right.\right. \\
& \text { - } \left.\left\|\left(T_{r_{m}}^{\left(G_{2}, \phi_{2}\right)}-I\right) A v_{m}\right\|^{2}\right\} \\
& \leq 2 \eta\left\{\frac{1}{2}\left\|\left(T_{r_{m}}^{\left(G_{2}, \phi_{2}\right)}-I\right) A v_{m}\right\|^{2}-\left\|\left(T_{r_{m}}^{\left(G_{2}, \phi_{2}\right)}-I\right) A v_{m}\right\|^{2}\right\} \\
& =-\eta\left\|\left(T_{r_{m}}^{\left(G_{2}, \phi_{2}\right)}-I\right) A v_{m}\right\|^{2} \text {. }
\end{aligned}
$$

Thus, we obtain

$$
\left.\left\|w_{m}-p\right\|^{2} \leq\left\|v_{m}-p\right\|^{2}+\eta(\eta L-1) \|\left(T_{r_{m}}^{\left(G_{2}, \phi_{2}\right)}-I\right) A v_{m}\right) \|^{2} .
$$


From the definition of $\eta$, we obtain

$$
\left\|w_{m}-p\right\| \leq\left\|v_{m}-p\right\| \leq\left\|x_{m}-p\right\| .
$$

Since $0<\sigma_{m} \rho<\|W\|^{-1}$, by Lemma 3, we get

$$
\left\|I-\sigma_{m} \rho W\right\| \leq 1-\sigma_{m} \rho \zeta .
$$

It follows that

$$
\begin{aligned}
\left\|x_{m+1}-p\right\|= & \left\|\sigma_{m} \gamma f\left(x_{m}\right)+b_{m} x_{m}+\left(\left(1-b_{m}\right) I-\sigma_{m} \rho W\right) w_{m}-p\right\| \\
= & \| \sigma_{m}\left(\gamma f\left(x_{m}\right)-\rho W p\right)+b_{m}\left(x_{m}-p\right) \\
& +\left(\left(1-b_{m}\right) I-\sigma_{m} \rho W\right)\left(w_{m}-p\right) \| \\
\leq & \left\|\sigma_{m}\left(\gamma f\left(x_{m}\right)-\rho W p\right)\right\|+b_{m}\left\|x_{m}-p\right\| \\
& +\left\|\left(\left(1-b_{m}\right) I-\sigma_{m} \rho W\right)\left(w_{m}-p\right)\right\| \\
\leq & \sigma_{m}\left\|\gamma f\left(x_{m}\right)-\gamma f(p)\right\|+\sigma_{m}\left\|\gamma f\left(x_{m}\right)-\rho W p\right\| \\
& +b_{m}\left\|x_{m}-p\right\|+\left(1-b_{m}-\sigma_{m} \rho \zeta\right)\left\|w_{m}-p\right\| \\
\leq & \sigma_{m} \gamma \tau\left\|x_{m}-p\right\|+b_{m}\left\|x_{m}-p\right\| \\
& +\left(1-b_{m}-\sigma_{m} \rho \zeta\right)\left\|x_{m}-p\right\|+\sigma_{m}\|\gamma f(p)-\rho W p\| \\
= & {\left[1-\sigma_{m}(\rho \zeta-\gamma \tau)\right]\left\|x_{m}-p\right\|+\sigma_{m}(\rho \zeta-\gamma \tau) \frac{\|\gamma f(p)-\rho W p\|}{\rho \zeta-\gamma \tau} . }
\end{aligned}
$$

Continuing in the same way, we see that

$$
\left\|x_{m+1}-p\right\| \leq \max \left\{\left\|x_{0}-p\right\|, \frac{\|\gamma f(p)-\rho W p\|}{\rho \zeta-\gamma \tau}\right\}, \quad m \geq 0, x_{0} \in C .
$$

Therefore, the sequence $\left\{x_{m}\right\}$ is bounded, and so are the sequences $\left\{v_{m}\right\}$, $\left\{w_{m}\right\},\left\{y_{m}\right\},\left\{z_{m}\right\},\left\{f\left(x_{m}\right)\right\}$ and $\left\{W\left(w_{m}\right)\right\}$.

Theorem 2 Let $p \in \mathcal{F}$ and $\left\{x_{m}\right\}$ be the sequence generated by Algorithm. Then

(a) $\lim _{m \rightarrow \infty}\left\|x_{m+1}-x_{m}\right\|=0$;

(b) Algorithm converges weakly to $p \in \mathcal{F}$.

Proof. (a) Consider $t_{m}=\left(x_{m+1}-b_{m} x_{m}\right) /\left(1-b_{m}\right)$. Since

$$
\begin{aligned}
& t_{m+1}-t_{m}=\frac{x_{m+2}-b_{m+1} x_{m+1}}{1-b_{m+1}}-\frac{x_{m+1}-b_{m} x_{m}}{1-b_{m}} \\
& =\frac{\sigma_{m+1} \gamma f\left(x_{m+1}\right)+\left(\left(1-b_{m+1}\right) I-\sigma_{m+1} \rho W\right) w_{m+1}}{1-b_{m+1}} \\
& -\frac{\sigma_{m} \gamma f\left(x_{m}\right)+\left(\left(1-b_{m}\right) I-\sigma_{m} \rho W\right) w_{m}}{1-b_{m}} \\
& =\frac{\sigma_{m+1}\left(\gamma f\left(x_{m+1}\right)-\rho W w_{m+1}\right)}{1-b_{m+1}}-\frac{\sigma_{m}\left(\gamma f\left(x_{m}\right)-\rho W w_{m}\right)}{1-b_{m}}+w_{m+1}-w_{m},
\end{aligned}
$$


we have

$$
\begin{aligned}
\left\|t_{m+1}-t_{m}\right\| \leq & \frac{\sigma_{m+1}}{1-b_{m+1}}\left\|\gamma f\left(x_{m+1}\right)-\rho W w_{m+1}\right\| \\
& +\frac{\sigma_{m}}{1-b_{m}}\left\|\gamma f\left(x_{m}\right)-\rho W w_{m}\right\|+\left\|w_{m+1}-w_{m}\right\| \\
\leq & \frac{\sigma_{m+1}}{1-b}\left\|\gamma f\left(x_{m+1}\right)-\rho W w_{m+1}\right\| \\
& +\frac{\sigma_{m}}{1-b}\left\|\gamma f\left(x_{m}\right)-\rho W w_{m}\right\|+\left\|w_{m+1}-w_{m}\right\| .
\end{aligned}
$$

Using Lemma 8, we can write

$$
\begin{aligned}
& \left\|w_{m+1}-w_{m}\right\|=\| T_{r_{m+1}}^{\left(G_{1}, \phi_{1}\right)}\left(v_{m+1}+\eta A^{*}\left(T_{r_{m+1}}^{\left(G_{2}, \phi_{2}\right)}-I\right) A v_{m+1}\right) \\
& -T_{r_{m}}^{\left(G_{1}, \phi_{1}\right)}\left(v_{m}+\eta A^{*}\left(T_{r_{m}}^{\left(G_{2}, \phi_{2}\right)}-I\right) A v_{m}\right) \| \\
& \leq \| T_{r_{m+1}}^{\left(G_{1}, \phi_{1}\right)}\left(v_{m+1}+\eta A^{*}\left(T_{r_{m+1}}^{\left(G_{2}, \phi_{2}\right)}-I\right) A v_{m+1}\right) \\
& -T_{r_{m+1}}^{\left(G_{1}, \phi_{1}\right)}\left(v_{m}+\eta A^{*}\left(T_{r_{m}}^{\left(G_{2}, \phi_{2}\right)}-I\right) A v_{m}\right) \| \\
& +\| T_{r_{m+1}}^{\left(G_{1}, \phi_{1}\right)}\left(v_{m}+\eta A^{*}\left(T_{r_{m}}^{\left(G_{2}, \phi_{2}\right)}-I\right) A v_{m}\right) \\
& -T_{r_{m}}^{\left(G_{1}, \phi_{1}\right)}\left(v_{m}+\eta A^{*}\left(T_{r_{m}}^{\left(G_{2}, \phi_{2}\right)}-I\right) A v_{m}\right) \| \\
& \leq\left\|\left(v_{m+1}+\eta A^{*}\left(T_{r_{m+1}}^{\left(G_{2}, \phi_{2}\right)}-I\right) A v_{m+1}\right)-\left(v_{m}+\eta A^{*}\left(T_{r_{m}}^{\left(G_{2}, \phi_{2}\right)}-I\right) A v_{m}\right)\right\| \\
& +\left|\frac{r_{m+1}-r_{m}}{r_{m+1}}\right| \| T_{r_{m+1}}^{\left(G_{1}, \phi_{1}\right)}\left(v_{m}+\eta A^{*}\left(T_{r_{m}}^{\left(G_{2}, \phi_{2}\right)}-I\right) A v_{m}\right) \\
& -\left(v_{m}+\eta A^{*}\left(T_{r_{m}}^{\left(G_{2}, \phi_{2}\right)}-I\right) A v_{m}\right) \| \\
& \leq\left\|v_{m+1}-v_{m}-\eta A^{*} A\left(v_{m+1}-v_{m}\right)\right\|+\eta\|A\|\left\|T_{r_{m+1}}^{\left(G_{2}, \phi_{2}\right)} A v_{m+1}-T_{r_{m}}^{\left(G_{2}, \phi_{2}\right)} A v_{m}\right\| \\
& +s_{m} \leq\left\{\left\|v_{m+1}-v_{m}\right\|^{2}-2 \eta\left\|A v_{m+1}-A v_{m}\right\|^{2}+\eta^{2}\|A\|^{4}\left\|v_{m+1}-v_{m}\right\|^{2}\right\}^{1 / 2} \\
& +\eta\|A\|\left\{\left\|A v_{m+1}-A v_{m}\right\|+\left|\frac{r_{m+1}-r_{m}}{r_{m+1}}\right|\left\|T_{r_{m+1}}^{\left(G_{2}, \phi_{2}\right)} A v_{m+1}-A v_{m+1}\right\|\right\}+s_{m} \\
& \leq\left(1-2 \eta\|A\|^{2}+\eta^{2}\|A\|^{4}\right)^{\frac{1}{2}}\left\|v_{m+1}-v_{m}\right\|+\eta\|A\|^{2}\left\|v_{m+1}-v_{m}\right\|+\eta\|A\| \omega_{m} \\
& +s_{m}=\left(1-\eta\|A\|^{2}\right)\left\|v_{m+1}-v_{m}\right\|+\eta\|A\|^{2}\left\|v_{m+1}-v_{m}\right\|+\eta\|A\| \omega_{m}+s_{m} \\
& =\left\|v_{m+1}-v_{m}\right\|+\eta\|A\| \omega_{m}+s_{m},
\end{aligned}
$$


where

$$
\omega_{m}=\left|\frac{r_{m+1}-r_{m}}{r_{m+1}}\right|\left\|T_{r_{m+1}}^{\left(G_{2}, \phi_{2}\right)} A v_{m+1}-A v_{m+1}\right\|
$$

and

$$
\begin{aligned}
s_{m}= & \left|\frac{r_{m+1}-r_{m}}{r_{m+1}}\right| \| T_{r_{m+1}}^{\left(G_{1}, \phi_{1}\right)}\left(v_{m}+\eta A^{*}\left(T_{r_{m}}^{\left(G_{2}, \phi_{2}\right)}-I\right) A v_{m}\right) \\
& -\left(v_{m}+\eta A^{*}\left(T_{r_{m}}^{\left(G_{2}, \phi_{2}\right)}-I\right) A v_{m}\right) \| .
\end{aligned}
$$

Further,

$$
\begin{aligned}
\left\|v_{m+1}-v_{m}\right\| & =\left\|U_{m+1} u_{m+1}-U_{m} u_{m}\right\| \\
& =\left\|U_{m+1} u_{m+1}-U_{m+1} u_{m}+U_{m+1} u_{m}-U_{m} u_{m}\right\| \\
& \leq\left\|U_{m+1} u_{m+1}-U_{m+1} u_{m}\right\|+\left\|U_{m+1} u_{m}-U_{m} u_{m}\right\| \\
& \leq\left\|u_{m+1}-u_{m}\right\|+\left\|U_{m+1} u_{m}-U_{m} u_{m}\right\| \\
& \leq\left\|y_{m+1}-y_{m}\right\|+\left\|U_{m+1} u_{m}-U_{m} u_{m}\right\| .
\end{aligned}
$$

Since,

$$
\begin{aligned}
&\left\|y_{m+1}-y_{m}\right\|=\| x_{m+1}+\eta A^{*}\left(V_{m+1} J_{\lambda, E_{2}}-I\right) A x_{m+1} \\
& \quad-x_{m}-\eta A^{*}\left(V_{m} J_{\lambda, E_{2}}-I\right) A x_{m} \| \\
& \leq\left\|x_{m+1}-x_{m}-\eta A^{*} A\left(x_{m+1}-x_{m}\right)\right\|+\eta\|A\|\left\|V_{m+1} z_{m+1}-V_{m} z_{m}\right\| \\
&=\left\{\left\|x_{m+1}-x_{m}\right\|^{2}-2 \eta\left\|A x_{m+1}-A x_{m}\right\|^{2}+\eta^{2}\|A\|^{4}\left\|x_{m+1}-x_{m}\right\|^{2}\right\}^{1 / 2} \\
&+\eta\|A\|\left\|V_{m+1} z_{m+1}-V_{m+1} z_{m}+V_{m+1} z_{m}-V_{m} z_{m}\right\| \\
& \leq\left(1-\eta\|A\|^{2}\right)\left\|x_{m+1}-x_{m}\right\| \\
&+\eta\|A\|\left(\left\|V_{m+1} z_{m+1}-V_{m+1} z_{m}\right\|+\left\|V_{m+1} z_{m}-V_{m} z_{m}\right\|\right) \\
& \leq\left(1-\eta\|A\|^{2}\right)\left\|x_{m+1}-x_{m}\right\|+\eta\|A\|\left(\left\|z_{m+1}-z_{m}\right\|+\left\|V_{m+1} z_{m}-V_{m} z_{m}\right\|\right) \\
& \leq\left(1-\eta\|A\|^{2}\right)\left\|x_{m+1}-x_{m}\right\| \\
&+\eta\|A\|\left(\left\|J_{\lambda, E_{2}} A x_{m+1}-J_{\lambda, E_{2}} A x_{m}\right\|+\left\|V_{m+1} z_{m}-V_{m} z_{m}\right\|\right) \\
& \leq\left(1-\eta\|A\|^{2}\right)\left\|x_{m+1}-x_{m}\right\|+\eta\|A\|\left(\left\|A x_{m+1}-A x_{m}\right\|+\left\|V_{m+1} z_{m}-V_{m} z_{m}\right\|\right) \\
& \leq\left\|x_{m+1}-x_{m}\right\|+\eta\|A\|\left\|V_{m+1} z_{m}-V_{m} z_{m}\right\|,
\end{aligned}
$$

we get

$$
\begin{aligned}
\left\|v_{m+1}-v_{m}\right\| \leq & \left\|x_{m+1}-x_{m}\right\|+\eta\|A\|\left\|V_{m+1} z_{m}-V_{m} z_{m}\right\| \\
& +\left\|U_{m+1} u_{m}-U_{m} u_{m}\right\| .
\end{aligned}
$$


Therefore,

$$
\begin{aligned}
& \left\|w_{m+1}-w_{m}\right\| \leq\left\|x_{m+1}-x_{m}\right\|+\eta\|A\|\left\|V_{m+1} z_{m}-V_{m} z_{m}\right\| \\
& +\eta\|A\| \omega_{m}+\left\|U_{m+1} u_{m}-U_{m} u_{m}\right\|+s_{m} \\
& =\left\|x_{m+1}-x_{m}\right\|+\eta\|A\|\left(\left\|V_{m+1} z_{m}-V_{m} z_{m}\right\|+\omega_{m}\right)+\left\|U_{m+1} u_{m}-U_{m} u_{m}\right\|+s_{m} .
\end{aligned}
$$

Substituting obtained estimations, we get

$$
\begin{aligned}
& \left\|t_{m+1}-t_{m}\right\|-\left\|x_{m+1}-x_{m}\right\| \\
& \leq \frac{\sigma_{m+1}}{1-b}\left\|\gamma f\left(x_{m+1}\right)-\rho W w_{m+1}\right\|+\frac{\sigma_{m}}{1-b}\left\|\gamma f\left(x_{m}\right)-\rho W w_{m}\right\| \\
& \quad+\eta\|A\|\left(\left\|V_{m+1} z_{m}-V_{m} z_{m}\right\|+\omega_{m}\right)+\left\|U_{m+1} u_{m}-U_{m} u_{m}\right\|+s_{m} .
\end{aligned}
$$

Further,

$$
\begin{aligned}
& \left\|U_{m+1} u_{m}-U_{m} u_{m}\right\|=\left\|U_{2}^{m+1} U_{1}^{m+1} u_{m}-U_{2}^{m} U_{1}^{m} u_{m}\right\| \\
& \leq\left\|U_{2}^{m+1} U_{1}^{m+1} u_{m}-U_{2}^{m+1} U_{1}^{m} u_{m}\right\|+\left\|U_{2}^{m+1} U_{1}^{m} u_{m}-U_{2}^{m} U_{1}^{m} u_{m}\right\| .
\end{aligned}
$$

It follows from the definition of $U_{i}^{m}$ that

$$
\begin{aligned}
& \left\|U_{1}^{m+1} u_{m}-U_{1}^{m} u_{m}\right\| \\
& \left.\left.\quad=\|\left(1-\eta_{m+1}^{1}\right) u_{m}+\eta_{m+1}^{1}\right) U_{1} u_{m}-\left(1-\eta_{m}^{1}\right) u_{m}+\eta_{m}^{1}\right) U_{1} u_{m} \| \\
& \leq\left|\eta_{m+1}^{1}-\eta_{m}^{1}\right|\left(\left\|u_{m}\right\|+\left\|U_{1} u_{m}\right\|\right) .
\end{aligned}
$$

Since $\lim _{m \rightarrow \infty}\left|\eta_{m+1}^{1}-\eta_{m}^{1}\right|=0$ for $i=1,2$ and the sequences $\left\{u_{m}\right\}$ and $\left\{U_{1} u_{m}\right\}$ are bounded, we get

$$
\lim _{m \rightarrow \infty}\left\|U_{1}^{m+1} u_{m}-U_{1}^{m} u_{m}\right\|=0
$$

Similarly,

$$
\left\|U_{2}^{m+1} U_{1}^{m} u_{m}-U_{2}^{m} U_{1}^{m} u_{m}\right\| \leq\left|\eta_{m+1}^{2}-\eta_{m}^{2}\right|\left(\left\|U_{1}^{m} u_{m}\right\|+\left\|U_{2}^{m} U_{1}^{m} u_{m}\right\|\right),
$$

from which it follows that

$$
\lim _{m \rightarrow \infty}\left\|U_{2}^{m+1} U_{1}^{m} u_{m}-U_{2}^{m} U_{1}^{m} u_{m}\right\|=0
$$

Therefore,

$$
\lim _{m \rightarrow \infty}\left\|U_{m+1} u_{m}-U_{m} u_{m}\right\|=0 .
$$

Using the similar reasoning, one can show that

$$
\lim _{m \rightarrow \infty}\left\|V_{m+1} u_{m}-V_{m} u_{m}\right\|=0 .
$$

Hence, due to (20), 21) and (B1),(B2), we get

$$
\lim _{m \rightarrow \infty}\left(\left\|t_{m+1}-t_{m}\right\|-\left\|x_{m+1}-x_{m}\right\|\right) \leq 0 .
$$


Thus, by Lemma 10, we conclude that $\lim _{m \rightarrow \infty}\left\|t_{m}-x_{m}\right\|=0$, which implies that

$$
\lim _{m \rightarrow \infty}\left\|x_{m+1}-x_{m}\right\|=0 .
$$

(b) To show the weak convergence of Algorithm, consider

$$
\begin{aligned}
& \left\|x_{m}-w_{m}\right\| \leq\left\|x_{m}-x_{m+1}\right\|+\left\|x_{m+1}-w_{m}\right\| \\
& \leq\left\|x_{m}-x_{m+1}\right\|+\left\|\sigma_{m} \gamma f\left(x_{m}\right)+b_{m} x_{m}+\left(\left(1-b_{m}\right) I-\sigma_{m} \rho W\right) w_{m}-w_{m}\right\| \\
& \leq\left\|x_{m}-x_{m+1}\right\|+\sigma_{m}\left\|\gamma f\left(x_{m}\right)-\rho W w_{m}\right\|+b_{m}\left\|x_{m}-w_{m}\right\| \\
& \leq\left\|x_{m}-x_{m+1}\right\|+\sigma_{m}\left(\left\|\gamma f\left(x_{m}\right)\right\|+\left\|\rho W w_{m}\right\|\right)+b_{m}\left\|x_{m}-w_{m}\right\| .
\end{aligned}
$$

That is,

$$
\left\|x_{m}-w_{m}\right\| \leq \frac{1}{1-b_{m}}\left\|x_{m}-x_{m+1}\right\|+\frac{\sigma_{m}}{1-b_{m}}\left(\left\|\gamma f\left(x_{m}\right)\right\|+\left\|\rho W w_{m}\right\|\right),
$$

which together with (B1) implies that

$$
\lim _{m \rightarrow \infty}\left\|x_{m}-w_{m}\right\|=0 .
$$

Now let us show that $\lim _{m \rightarrow \infty}\left\|x_{m}-v_{m}\right\|=0$. Using the non-expansivity of averaged mappings, we can write

$$
\begin{aligned}
&\left\|v_{m}-p\right\|^{2}=\left\|U_{m} J_{\lambda, E_{1}}\left(x_{m}+\eta A^{*}\left(V_{m} J_{\lambda, E_{2}}-I\right) A x_{m}\right)-p\right\|^{2} \\
&=\left\|U_{m} J_{\lambda, E_{1}}\left(x_{m}+\eta A^{*}\left(V_{m} J_{\lambda, E_{2}}-I\right) A x_{m}\right)-U_{m} J_{\lambda, E_{1}} p\right\|^{2} \\
&=\left\|x_{m}+\eta A^{*}\left(V_{m} J_{\lambda, E_{2}}-I\right) A x_{m}-p\right\|^{2} \\
& \leq\left\langle v_{m}-p, x_{m}+\eta A^{*}\left(V_{m} J_{\lambda, E_{2}}-I\right) A x_{m}-p\right\rangle \\
&= \frac{1}{2}\left\{\left\|v_{m}-p\right\|^{2}+\left\|x_{m}+\eta A^{*}\left(V_{m} J_{\lambda, E_{2}}-I\right) A x_{m}-p\right\|^{2}\right. \\
&\left.-\left\|\left(v_{m}-p\right)-\left(x_{m}+\eta A^{*}\left(V_{m} J_{\lambda, E_{2}}-I\right) A x_{m}-p\right)\right\|^{2}\right\} \\
&= \frac{1}{2}\left\{\left\|v_{m}-p\right\|^{2}+\left\|x_{m}-p\right\|^{2}+\eta(L \eta-1)\left(V_{m} J_{\lambda, E_{2}}-I\right) A x_{m} \|^{2}\right. \\
&\left.-\left\|v_{m}-x_{m}-\eta A^{*}\left(V_{m} J_{\lambda, E_{2}}-I\right) A x_{m}\right\|^{2}\right\} \\
&= \frac{1}{2}\left\{\left\|v_{m}-p\right\|^{2}+\left\|x_{m}-p\right\|^{2}-\left[\left\|v_{m}-x_{m}\right\|^{2}\right.\right. \\
&\left.\left.+\eta^{2}\left\|A^{*}\left(V_{m} J_{\lambda, E_{2}}-I\right) A x_{m}\right\|^{2}-2 \eta\left\langle v_{m}-x_{m}, A^{*}\left(V_{m} J_{\lambda, E_{2}}-I\right) A x_{m}\right\rangle\right]\right\} \\
&= \frac{1}{2}\left\{\left\|v_{m}-p\right\|^{2}+\left\|x_{m}-p\right\|^{2}-\left\|v_{m}-x_{m}\right\|^{2}\right. \\
&\left.+2 \eta\left\|A\left(v_{m}-x_{m}\right)\right\|\left\|\left(V_{m} J_{\lambda, E_{2}}-I\right) A x_{m}\right\|\right\} .
\end{aligned}
$$


Hence,

$$
\begin{aligned}
\left\|v_{m}-p\right\|^{2} \leq\left\|x_{m}-p\right\|^{2}- & \left\|v_{m}-x_{m}\right\|^{2} \\
& +2 \eta\left\|A\left(v_{m}-x_{m}\right)\right\|\left\|\left(V_{m} J_{\lambda, E_{2}}-I\right) A x_{m}\right\| .
\end{aligned}
$$

Now we consider $\left\|x_{m+1}-p\right\|^{2}$. Using (15) and (16), we can write

$$
\begin{aligned}
&\left\|x_{m+1}-p\right\|^{2}=\left\|\sigma_{m} \gamma f\left(x_{m}\right)+b_{m} x_{m}+\left(\left(1-b_{m}\right) I-\sigma_{m} \rho W\right) w_{m}-p\right\|^{2} \\
&=\left\|\sigma_{m}\left(\gamma f\left(x_{m}\right)-\rho W p\right)+b_{m}\left(x_{m}-w_{m}\right)+\left(I-\sigma_{m} \rho W\right)\left(w_{m}-p\right)\right\|^{2} \\
& \leq\left\|b_{m}\left(x_{m}-w_{m}\right)+\left(I-\sigma_{m} \rho W\right)\left(w_{m}-p\right)\right\|^{2} \\
&+2 \sigma_{m}\left\langle\gamma f\left(x_{m}\right)-\rho W p, x_{m+1}-p\right\rangle \\
& \leq {\left[\left(1-\sigma_{m} \rho \zeta\right)\left\|w_{m}-p\right\|+b_{m}\left\|x_{m}-w_{m}\right\|^{2}\right] } \\
&+2 \sigma_{m}\left\|\gamma f\left(x_{m}\right)-\rho W p\right\|\left\|x_{m+1}-p\right\| \\
& \leq\left(1-\sigma_{m} \rho \zeta\right)^{2}\left\|w_{m}-p\right\|^{2}+b_{m}^{2}\left\|x_{m}-w_{m}\right\|^{2} \\
&+2 b_{m}\left(1-\sigma_{m} \rho \zeta\right)\left\|w_{m}-p\right\|\left\|x_{m}-w_{m}\right\| \\
&+2 \sigma_{m}\left\|\gamma f\left(x_{m}\right)-\rho W p\right\|\left\|x_{m+1}-p\right\| .
\end{aligned}
$$

Using (??), we obtain

$$
\begin{aligned}
& \left.\left\|x_{m+1}-p\right\|^{2} \leq\left(1-\sigma_{m} \rho \zeta\right)^{2}\left[\left\|v_{m}-p\right\|^{2}+\eta(\eta L-1) \|\left(T_{r_{m}}^{\left(G_{2}, \phi_{2}\right)}-I\right) A v_{m}\right) \|^{2}\right] \\
& \quad+b_{m}^{2}\left\|x_{m}-w_{m}\right\|^{2}+2 b_{m}\left(1-\sigma_{m} \rho \zeta\right)\left\|w_{m}-p\right\|\left\|x_{m}-w_{m}\right\| \\
& \quad+2 \sigma_{m}\left\|\gamma f\left(x_{m}\right)-\rho W p\right\|\left\|x_{m+1}-p\right\| \\
& \left.\leq\left(1-\sigma_{m} \rho \zeta\right)^{2}\left[\left\|x_{m}-p\right\|^{2}+\eta(\eta L-1) \|\left(T_{r_{m}}^{\left(G_{2}, \phi_{2}\right)}-I\right) A v_{m}\right) \|^{2}\right] \\
& \quad+b_{m}^{2}\left\|x_{m}-w_{m}\right\|^{2}+2 b_{m}\left(1-\sigma_{m} \rho \zeta\right)\left\|w_{m}-p\right\|\left\|x_{m}-w_{m}\right\| \\
& \quad+2 \sigma_{m}\left\|\gamma f\left(x_{m}\right)-\rho W p\right\|\left\|x_{m+1}-p\right\| .
\end{aligned}
$$

Therefore,

$$
\begin{aligned}
& \left.\left(1-\sigma_{m} \rho \zeta\right)^{2} \eta(1-\eta L) \|\left(T_{r_{m}}^{\left(G_{2}, \phi_{2}\right)}-I\right) A v_{m}\right) \|^{2} \\
& \leq b_{m}^{2}\left\|x_{m}-w_{m}\right\|^{2}+2 b_{m}\left(1-\sigma_{m} \rho \zeta\right)\left\|w_{m}-p\right\|\left\|x_{m}-w_{m}\right\| \\
& \quad+2 \sigma_{m}\left\|\gamma f\left(x_{m}\right)-\rho W p\right\|\left\|x_{m+1}-p\right\| \\
& \quad+\left(1-\sigma_{m} \rho \zeta\right)^{2}\left\|x_{m}-p\right\|^{2}-\left\|x_{m+1}-p\right\|
\end{aligned}
$$

which gives

$$
\begin{aligned}
& \left.\left(1-\sigma_{m} \rho \zeta\right)^{2} \eta(1-\eta L) \|\left(T_{r_{m}}^{\left(G_{2}, \phi_{2}\right)}-I\right) A v_{m}\right) \|^{2} \\
& \leq\left(\sigma_{m} \rho \zeta\right)^{2}\left\|x_{m}-p\right\|^{2}+2 b_{m}\left(1-\sigma_{m} \rho \zeta\right)\left\|w_{m}-p\right\|\left\|x_{m}-w_{m}\right\| \\
& \quad+2 \sigma_{m}\left\|\gamma f\left(x_{m}\right)-\rho W p\right\|\left\|x_{m+1}-p\right\|+b_{m}^{2}\left\|x_{m}-w_{m}\right\|^{2} \\
& \quad-2 \sigma_{m} \rho \zeta\left\|x_{m}-p\right\|^{2}+\left\|x_{m}-x_{m+1}\right\|\left(\left\|x_{m}-p\right\|+\left\|x_{m+1}-p\right\|\right) .
\end{aligned}
$$


Further, using (19), we can write

$$
\begin{aligned}
\left\|x_{m+1}-p\right\|^{2} \leq & \left(1-\sigma_{m} \rho \zeta\right)^{2}\left[\left\|x_{m}-p\right\|^{2}+\eta(\eta L-1) \|\left(V_{m} J_{\lambda, E_{2}}-I\right) A x_{m}\right) \|^{2} \\
& \left.\left.-\left\|z_{m}-A x_{m}\right\|^{2}+\eta(\eta L-1) \|\left(T_{r_{m}}^{\left(G_{2}, \phi_{2}\right)}-I\right) A v_{m}\right) \|^{2}\right] \\
& +b_{m}^{2}\left\|x_{m}-w_{m}\right\|^{2}+2 b_{m}\left(1-\sigma_{m} \rho \zeta\right)\left\|w_{m}-p\right\|\left\|x_{m}-w_{m}\right\| \\
& +2 \sigma_{m}\left\|\gamma f\left(x_{m}\right)-\rho W p\right\|\left\|x_{m+1}-p\right\| .
\end{aligned}
$$

Since due to (B3), (22) and (23), we get

$$
\left.\lim _{m \rightarrow \infty} \|\left(T_{r_{m}}^{\left(G_{2}, \phi_{2}\right)}-I\right) A v_{m}\right) \|=0,
$$

we obtain

$$
\begin{aligned}
\left\|x_{m+1}-p\right\|^{2} \leq & \left(1-\sigma_{m} \rho \zeta\right)^{2}\left[\left\|x_{m}-p\right\|^{2}+\eta(\eta L-1)\left\|\left(V_{m} z_{m}-A x_{m}\right)\right\|^{2}\right. \\
& \left.-\left\|z_{m}-A x_{m}\right\|^{2}\right]+b_{m}^{2}\left\|x_{m}-w_{m}\right\|^{2} \\
& +2 b_{m}\left(1-\sigma_{m} \rho \zeta\right)\left\|w_{m}-p\right\|\left\|x_{m}-w_{m}\right\| \\
& +2 \sigma_{m}\left\|\gamma f\left(x_{m}\right)-\rho W p\right\|\left\|x_{m+1}-p\right\| .
\end{aligned}
$$

Hence,

$$
\begin{aligned}
& \left(1-\sigma_{m} \rho \zeta\right)^{2}\left[\eta(\eta L-1)\left\|\left(V_{m} z_{m}-A x_{m}\right)\right\|^{2}-\left\|z_{m}-A x_{m}\right\|^{2}\right] \\
& \leq b_{m}^{2}\left\|x_{m}-w_{m}\right\|^{2}+2 b_{m}\left(1-\sigma_{m} \rho \zeta\right)\left\|w_{m}-p\right\|\left\|x_{m}-w_{m}\right\| \\
& \quad+2 \sigma_{m}\left\|\gamma f\left(x_{m}\right)-\rho W p\right\|\left\|x_{m+1}-p\right\|+\left(1-\sigma_{m} \rho \zeta\right)^{2}\left\|x_{m}-p\right\|^{2} \\
& \quad-\left\|x_{m+1}-p\right\|^{2} .
\end{aligned}
$$

Thus, from (23) and (B2), we get

$$
\lim _{m \rightarrow \infty}\left[\left\|V_{m} z_{m}-A x_{m}\right\|^{2}+\left\|z_{m}-A x_{m}\right\|^{2}\right]=0,
$$

which means that

$$
\lim _{m \rightarrow \infty}\left\|V_{m} z_{m}-A x_{m}\right\|=0 .
$$

Due to (24), we have

$$
\begin{aligned}
\left\|x_{m+1}-p\right\|^{2} \leq & \left(1-\sigma_{m} \rho \zeta\right)^{2}\left[\left\|x_{m}-p\right\|^{2}-\left\|v_{m}-x_{m}\right\|^{2}\right. \\
& \left.+2 \eta\left\|A\left(v_{m}-x_{m}\right)\right\|\left\|V_{m} z_{m}-A x_{m}\right\|\right]+b_{m}^{2}\left\|x_{m}-w_{m}\right\|^{2} \\
& +2 b_{m}\left(1-\sigma_{m} \rho \zeta\right)\left\|w_{m}-p\right\|\left\|x_{m}-w_{m}\right\| \\
& +2 \sigma_{m}\left\|\gamma f\left(x_{m}\right)-\rho W p\right\|\left\|x_{m+1}-p\right\|,
\end{aligned}
$$


and therefore,

$$
\begin{aligned}
& \left(1-\sigma_{m} \rho \zeta\right)^{2}\left\|v_{m}-x_{m}\right\|^{2} \\
& \leq 2 \eta\left(1-\sigma_{m} \rho \zeta\right)^{2}\left\|A\left(v_{m}-x_{m}\right)\right\|\left\|V_{m} z_{m}-A x_{m}\right\| \\
& \quad+b_{m}^{2}\left\|x_{m}-w_{m}\right\|^{2}+2 b_{m}\left(1-\sigma_{m} \rho \zeta\right)\left\|w_{m}-p\right\|\left\|x_{m}-w_{m}\right\| \\
& \quad+2 \sigma_{m}\left\|\gamma f\left(x_{m}\right)-\rho W p\right\|\left\|x_{m+1}-p\right\|+\left(\sigma_{m} \gamma \zeta\right)^{2}\left\|x_{m}-p\right\|^{2} \\
& \quad-2 \sigma_{m} \rho \zeta\left\|x_{m}-p\right\|^{2}+\left\|x_{m+1}-x_{m}\right\|\left(\left\|x_{m}-p\right\|+\left\|x_{m+1}-p\right\|\right) .
\end{aligned}
$$

Thus, from (B3), (23) and (26), we get

$$
\lim _{m \rightarrow \infty}\left\|v_{m}-x_{m}\right\|=0
$$

Now, we can write

$$
\begin{aligned}
\left\|x_{m}-U_{m} u_{m}\right\| \leq & \left\|x_{m}-x_{m+1}\right\|+\left\|x_{m+1}-U_{m} u_{m}\right\| \\
\leq & \left\|x_{m}-x_{m+1}\right\|+\| \sigma_{m} \gamma f\left(x_{m}\right)+b_{m} x_{m} \\
& +\left(\left(I-b_{m}\right)-\sigma_{m} \rho W\right) w_{m}-U_{m} u_{m} \| \\
\leq & \left\|x_{m}-x_{m+1}\right\|+\sigma_{m}\left\|\gamma f\left(x_{m}\right)-\rho W w_{m}\right\| \\
& +b_{m}\left\|x_{m}-u_{m} u_{m}\right\| \\
\leq & \left\|x_{m}-x_{m+1}\right\|+\sigma_{m}\left(\left\|\gamma f\left(x_{m}\right)\right\|+\left\|\rho W w_{m}\right\|\right) \\
& +b_{m}\left\|x_{m}-u_{m} u_{m}\right\|,
\end{aligned}
$$

that is

$$
\begin{aligned}
\left\|x_{m}-U_{m} u_{m}\right\| \leq & \frac{1}{1-b_{m}}\left\|x_{m}-x_{m+1}\right\| \\
& +\frac{\sigma_{m}}{1-b_{m}}\left(\left\|\gamma f\left(x_{m}\right)\right\|+\left\|\rho W w_{m}\right\|\right),
\end{aligned}
$$

which together with (B1) implies that

$$
\lim _{m \rightarrow \infty}\left\|x_{m}-U_{m} u_{m}\right\|=0 .
$$

From (27), we get

$$
\left\|x_{m+1}-v_{m}\right\| \leq\left\|x_{m+1}-x_{m}\right\|+\left\|x_{m}-v_{m}\right\| \rightarrow 0 \quad \text { as } \quad m \rightarrow \infty .
$$

From 22, (23) and $(29)$, we get

$$
\left\|w_{m}-v_{m}\right\| \leq\left\|w_{m}-x_{m}\right\|+\left\|x_{m}-x_{m+1}\right\|+\left\|x_{m+1}-v_{m}\right\| \rightarrow 0
$$

as $m \rightarrow \infty$. Further,

$$
\begin{aligned}
\left\|x_{m}-U_{m} x_{m}\right\| & \leq\left\|x_{m}-U_{m} u_{m}\right\|+\left\|U_{m} u_{m}-U_{m} w_{m}\right\|+\left\|U_{m} w_{m}-U_{m} x_{m}\right\| \\
& \leq\left\|x_{m}-U_{m} u_{m}\right\|+\left\|v_{m}-U_{m} w_{m}\right\|+\left\|w_{m}-x_{m}\right\| .
\end{aligned}
$$


From here it follows that

$$
\lim _{n \rightarrow \infty}\left\|x_{m}-U_{m} w_{m}\right\|=0 .
$$

Finally, using equation (28) and (30), we get

$$
\left\|x_{m}-U_{m} x_{m}\right\| \leq\left\|x_{m}-U_{m} u_{m}\right\|+\left\|v_{m}-U_{m} w_{m}\right\|+\left\|w_{m}-x_{m}\right\| \rightarrow 0
$$

as $m \rightarrow \infty$.

Theorem 3 The sequence $\left\{x_{m}\right\}$ generated by Algorithm strongly converges to $p^{*}$, which is the unique solution to the variational inequality (18).

Proof. First, we show that the solution of the variational inequality problem (18) is unique. Suppose towards a contradiction that $\hat{p}$ and $\tilde{p}$ are two different solutions to variational inequality problem (18). Then

$$
\langle(\rho W-\gamma f) \tilde{p}, \tilde{p}-\hat{p}\rangle \leq 0 \quad \text { and } \quad\langle(\rho W-\gamma f) \hat{p}, \hat{p}-\tilde{p}\rangle \leq 0 .
$$

Adding the above two inequalities, we get

$$
\langle(\rho W-\gamma f) \tilde{p}-(\rho W-\gamma f) \hat{p}, \tilde{p}-\hat{p}\rangle \leq 0 .
$$

According to (B1), $\rho \zeta>\gamma \tau$. Thus, by Lemma 5, we get

$$
\langle(\rho W-\gamma f) \tilde{p}-(\rho W-\gamma f) \hat{p}, \tilde{p}-\hat{p}\rangle \geq(\rho \zeta-\gamma \tau)\|\tilde{p}-\hat{p}\|^{2}>0,
$$

which leads to the contradiction. Hence, the variational inequality problem (18) has a unique solution $p^{*} \in \mathcal{F}$.

Now, we show that

$$
\left.\lim _{m \rightarrow \infty} \sup \left\langle(\rho W-\gamma f) p^{*}, p^{*}-x_{m}\right)\right\rangle \leq 0
$$

Due to the boundedness of $\left\{x_{m}\right\}$, there exists a subsequence $\left\{x_{m_{j}}\right\}$ of $\left\{x_{m}\right\}$ such that $x_{m_{j}} \rightarrow \bar{p}$ as $j \rightarrow \infty$ and

$$
\begin{aligned}
& \left.\lim _{m \rightarrow \infty} \sup \left\langle(\rho W-\gamma f) p^{*}, p^{*}-x_{m}\right)\right\rangle \\
& \left.=\lim _{m \rightarrow \infty} \sup \left\langle(\rho W-\gamma f) p^{*}, p^{*}-x_{m_{j}}\right)\right\rangle .
\end{aligned}
$$

Since $\left\|x_{m}-y_{m}\right\| \rightarrow 0$ as $m \rightarrow \infty$, we have $y_{m_{j}} \rightarrow \bar{p}$. Note that $\left\{\eta_{m}^{i}\right\}$ is bounded for $i=1,2$. We can assume that $\eta_{m_{j}}^{i} \rightarrow \eta_{\infty}^{i}$ as $j \rightarrow \infty$ where $0<\eta_{\infty}^{i}<1$ for $i=1,2$.

Define

$$
U_{i}^{\infty}=\left(1-\eta_{\infty}^{i}\right) I+\eta_{\infty}^{i} U_{i}, \quad i=1,2
$$


Then we have

$$
\operatorname{Fix}\left(U_{i}^{\infty}\right)=\operatorname{Fix}\left(U_{i}\right), \quad i=1,2 .
$$

Furthermore, since

$$
\operatorname{Fix}\left(U_{1}^{\infty}\right) \cap \operatorname{Fix}\left(U_{2}^{\infty}\right)=\operatorname{Fix}\left(U_{1}\right) \operatorname{Fix}\left(U_{2}\right)=\emptyset .
$$

and $U_{i}^{\infty}$ is $\eta_{\infty}^{i}$-averaged for $i=1,2$, by Lemma 1 , we get

$$
\operatorname{Fix}\left(U_{2}^{\infty} U_{1}^{\infty}\right)=\operatorname{Fix}\left(U_{1}^{\infty}\right) \operatorname{Fix}\left(U_{2}^{\infty}\right)=\operatorname{Fix}\left(U_{1}\right) \cap \operatorname{Fix}\left(U_{2}\right) .
$$

Note that

$$
\left\|U_{i}^{m_{j}} u-U_{i}^{\infty} u\right\| \leq\left|\eta_{m_{j}}^{i}-\eta_{\infty}^{i}\right|\left(\|u\|+\left\|U_{i} u\right\|\right)
$$

Hence,

$$
\lim _{j \rightarrow \infty} \sup _{u \in \mathcal{B}}\left\|U_{i}^{m_{j}} u-U_{i}^{\infty} u\right\|=0,
$$

where $\mathcal{B}$ is an arbitrary bounded subset of $\mathcal{H}_{1}$. Also, we have

$$
\begin{aligned}
\left\|x_{m_{j}}-U_{2}^{\infty} U_{1}^{\infty} x_{m_{j}}\right\| \leq & \left\|x_{m_{j}}-U_{2}^{m_{j}} U_{1}^{m_{j}} x_{m_{j}}\right\|+\left\|U_{2}^{m_{j}} U_{1}^{m_{j}} x_{m_{j}}-U_{2}^{\infty} U_{1}^{m_{j}} x_{m_{j}}\right\| \\
& +\left\|U_{2}^{\infty} U_{1}^{m_{j}} x_{m_{j}}-U_{2}^{\infty} U_{1}^{\infty} x_{m_{j}}\right\| \\
\leq & \left\|x_{m_{j}}-U_{2}^{m_{j}} U_{1}^{m_{j}} x_{m_{j}}\right\|+\left\|U_{2}^{m_{j}} U_{1}^{m_{j}} x_{m_{j}}-U_{2}^{\infty} U_{1}^{m_{j}} x_{m_{j}}\right\| \\
& +\left\|U_{1}^{m_{j}} x_{m_{j}}-U_{1}^{\infty} x_{m_{j}}\right\| \\
\leq & \left\|x_{m_{j}}-U_{2}^{m_{j}} U_{1}^{m_{j}} x_{m_{j}}\right\|+\sup _{x \in \mathcal{B}^{\prime}}\left\|U_{2}^{m_{j}} x-U_{2}^{\infty} x\right\| \\
& +\sup _{x \in \mathcal{B}^{\prime \prime}}\left\|U_{1}^{m_{j}} x-U_{1}^{\infty} x\right\|,
\end{aligned}
$$

where $\mathcal{B}^{\prime}$ is a bounded subset including $\left\{U_{1}^{m_{j}} x_{m_{j}}\right\}$, and $\mathcal{B}^{\prime \prime}$ is a bounded subset including $\left\{x_{m_{j}}\right\}$. It follows that

$$
\lim _{j \rightarrow \infty}\left\|x_{m_{j}}-U_{2}^{\infty} U_{1}^{\infty} x_{m_{j}}\right\|=0
$$

Thus, by Lemma 2, we have

$$
\bar{p} \in \operatorname{Fix}\left(U_{2}^{\infty} U_{1}^{\infty}\right)=\operatorname{Fix}\left(U_{1}\right) \cap \operatorname{Fix}\left(U_{2}\right) .
$$

Now, $y_{m_{j}} \rightarrow \bar{p}, u_{m_{j}} \rightarrow \bar{p}, A x_{m} \rightarrow A \bar{p}$, and $z_{m_{j}} \rightarrow A \bar{p}$. Using the above arguments, we can show that

$$
\lim _{j \rightarrow \infty}\left\|z_{m_{j}}-V_{2}^{\infty} V_{1}^{\infty} z_{m_{j}}\right\|=0
$$

Since $V_{2}^{\infty} V_{1}^{\infty}$ is non-expansive, by Lemma 2 we get

$$
A \bar{p} \in \operatorname{Fix}\left(V_{2}^{\infty} V_{1}^{\infty}\right)=\operatorname{Fix}\left(V_{1}\right) \cap \operatorname{Fix}\left(V_{2}\right) .
$$


Next, we show that $\bar{p} \in \operatorname{SOL}\left(E_{1}\right)$ and $A \bar{p} \in \operatorname{SOL}\left(E_{2}\right)$. Since $y_{m_{j}} \rightarrow \bar{p}$,

$$
\lim _{m \rightarrow \infty}\left\|u_{m}-y_{m}\right\|=\lim _{m \rightarrow \infty}\left\|J_{\lambda, E_{1}} y_{m}-y_{m}\right\|=0
$$

and $J_{\lambda, E_{1}}$ is non-expansive, by Lemma 2 , we get $\bar{p}=J_{\lambda, E_{1}} \bar{p}$, i.e., $\bar{p} \in$ $\operatorname{SOL}\left(E_{1}\right)$. Also, since $z_{m_{j}} \rightarrow A \bar{p}$,

$$
\lim _{m \rightarrow \infty}\left\|z_{m}-A x_{m}\right\|=\lim _{m \rightarrow \infty}\left\|J_{\lambda, E_{2}} A x_{m}-A x_{m}\right\|=0,
$$

and by Lemma 2, we get $A \bar{p}=J_{\lambda, E_{2}} A \bar{p}$, i.e., $A \bar{p} \in \operatorname{SOL}\left(E_{2}\right)$. Therefore, $\bar{p} \in \Omega$.

Now, we show that $\bar{p} \in \mathcal{S}$. First, we will show that $\bar{p} \in \operatorname{GEP}\left(G_{1}, \phi_{1}\right)$. Since $w_{m}=T_{r_{m}}^{\left(G_{1}, \phi_{1}\right)} v_{m}$, we have

$$
G_{1}\left(w_{m}, y\right)+\phi_{1}\left(w_{m}, y\right)+\frac{1}{r_{m}}\left\langle y-w_{m}, w_{m}-v_{m}\right\rangle \geq 0, \quad y \in C .
$$

It follows from the monotonicity of $G_{1}$ that

$$
\phi_{1}\left(w_{m}, y\right)+\frac{1}{r_{m}}\left\langle y-w_{m}, w_{m}-v_{m}\right\rangle \geq G_{1}\left(w_{m}, y\right),
$$

and hence, replacing $m$ by $m_{i}$, we get

$$
\phi_{1}\left(w_{m_{i}}, y\right)+\left\langle y-w_{m_{i}}, \frac{w_{m_{i}}-v_{m_{i}}}{r_{m_{i}}}\right\rangle \geq G_{1}\left(w_{m_{i}}, y\right) .
$$

Since $\left\|w_{m}-v_{m}\right\| \rightarrow 0$, we have $w_{m_{i}} \rightarrow \bar{p}$ and $\frac{w_{m_{i}}-v_{m_{i}}}{r_{m_{i}}} \rightarrow 0$. It follows from (iii) in Assumptions 1 , that $0 \geq G_{1}\left(w_{m}, y\right)$ for any $\bar{p} \in C$. For any $t$ with $0<t \leq 1$ and $y \in C$, let $y_{t}=t y+(1-t) \bar{p}$. Since $y, \bar{p} \in C$, we have $y_{t} \in C$, and hence, $G_{1}\left(y_{t}, \bar{p}\right) \leq 0$. Thus, due to (i) and (iv) in Assumptions 1, we obtain

$$
\begin{aligned}
0 & =G_{1}\left(y_{t}, y_{t}\right)+\phi_{1}\left(y_{t}, y_{t}\right) \\
& \leq t\left[G_{1}\left(y_{t}, y\right)+\phi_{1}\left(y_{t}, y\right)\right]+(1-t)\left[G_{1}\left(y_{t}, \bar{p}\right)+\phi_{1}\left(y_{t}, \bar{p}\right)\right] \\
& \leq t\left[G_{1}\left(y_{t}, y\right)+\phi_{1}\left(y_{t}, y\right)\right]+(1-t)\left[G_{1}\left(\bar{p}, y_{t}\right)+\phi_{1}\left(\bar{p}, y_{t}\right)\right] \\
& \leq t\left[G_{1}\left(y_{t}, y\right)+\phi_{1}\left(y_{t}, y\right)\right] .
\end{aligned}
$$

Therefore, $0 \leq G_{1}\left(y_{t}, y\right)+\phi_{1}\left(y_{t}, y\right)$. From Assumptions 1 (iii), we have $0 \leq G_{1}(\bar{p}, y)+\phi_{1}(\bar{p}, y)$. This implies that $\bar{p} \in \operatorname{GEP}\left(G_{1}, \phi_{1}\right)$.

Next, we show that $A \bar{p} \in \operatorname{GEP}\left(G_{2}, \phi_{2}\right)$. Since $\left\|w_{m}-v_{m}\right\| \rightarrow 0, \| v_{m}-$ $x_{m} \| \rightarrow 0$, we get $w_{m} \rightarrow \bar{p}$ as $m \rightarrow \infty$ and $\left\{x_{m}\right\}$ is bounded, there exists a subsequence $\left\{x_{m_{i}}\right\}$ such that $x_{m_{i}} \rightarrow \bar{p}$, and since $A$ is bounded linear operator, $A x_{m_{i}} \rightarrow A \bar{p}$. 
Now, set $q_{m_{i}}=A x_{m_{i}}-T_{r_{m_{i}}}^{\left(G_{2}, \phi_{2}\right)} A x_{m_{i}}$. It follows from (25), that

$$
\lim _{i \rightarrow \infty} q_{m_{i}}=0 \quad \text { and } \quad A x_{m_{i}}-q_{m_{i}}=T_{r_{m_{i}}}^{\left(G_{2}, \phi_{2}\right)} A x_{m_{i}}
$$

Therefore, from Lemma 7, we have

$$
\begin{aligned}
& G_{2}\left(A x_{m_{i}}-q_{m_{i}}, \tilde{\bar{p}}\right)+\phi_{2}\left(A x_{m_{i}}-q_{m_{i}}, \tilde{\bar{p}}\right) \\
& \quad+\frac{1}{r_{m_{i}}}\left\langle\tilde{\bar{p}}-\left(A x_{m_{i}}-q_{m_{i}}\right),\left(A x_{m_{i}}-q_{m_{i}}\right)-A x_{m_{i}}\right\rangle \geq 0
\end{aligned}
$$

for all $\tilde{\bar{p}} \in Q$. Since $G_{2}$ and $\phi_{2}$ are upper semi continuous, taking limit superior in (31) as $i \rightarrow \infty$ and using condition (B3), we obtain

$$
G_{2}(A \bar{p}, \tilde{\bar{p}})+\phi_{2}(A \bar{p}, \tilde{\bar{p}}) \geq 0, \quad \tilde{\bar{p}} \in Q
$$

which implies that $A \bar{p} \in \operatorname{GEP}\left(G_{2}, \phi_{2}\right)$ and hence, $\bar{p} \in \mathcal{S}$. Therefore, $\bar{p} \in \mathcal{F}$. Thus,

$$
\lim _{j \rightarrow \infty}\left\langle(\rho W-\gamma f) p^{*}, p^{*}-x_{m_{j}}\right\rangle \leq\left\langle(\rho W-\gamma f) p^{*}, p^{*}-\bar{p}\right\rangle \leq 0
$$

Finally, we show that $x_{m} \rightarrow p^{*}$ as $m \rightarrow \infty$. From (15) and (17), we have

$$
\begin{aligned}
\left\|x_{m+1}-p^{*}\right\|^{2}= & \left\|\sigma_{m} \gamma f\left(x_{m}\right)+b_{m} x_{m}+\left(\left(1-b_{m}\right) I-\sigma_{m} \rho W\right) w_{m}-p^{*}\right\|^{2} \\
= & \| \sigma_{m}\left(\gamma f\left(x_{m}\right)-\rho W p^{*}\right)+b_{m}\left(x_{m}-p^{*}\right) \\
& +\left(\left(1-b_{m}\right) I-\sigma_{m} \rho W\right)\left(w_{m}-p^{*}\right) \|^{2} \\
\leq & \left\|b_{m}\left(x_{m}-p^{*}\right)+\left(\left(1-b_{m}\right) I-\sigma_{m} \rho W\right)\left(w_{m}-p^{*}\right)\right\|^{2} \\
& +2 \sigma_{m}\left\langle\gamma f\left(x_{m}\right)-\rho W p^{*}, x_{m+1}-p^{*}\right\rangle \\
\leq & {\left[\left(1-b_{m}-\sigma_{m} \rho \zeta\right)\left\|w_{m}-p^{*}\right\|+b_{m}\left\|x_{m}-p^{*}\right\|\right]^{2} } \\
& +2 \sigma_{m}\left\langle\gamma f\left(x_{m}\right)-\gamma f\left(p^{*}\right), x_{m+1}-p^{*}\right\rangle \\
& +2 \sigma_{m}\left\langle\gamma f\left(p^{*}\right)-\rho W p^{*}, x_{m+1}-p^{*}\right\rangle \\
\leq & {\left[\left(1-b_{m}-\sigma_{m} \rho \zeta\right)\left\|x_{m}-p^{*}\right\|+b_{m}\left\|x_{m}-p^{*}\right\|\right]^{2} } \\
& +2 \sigma_{m} \gamma \tau\left\|x_{m}-p^{*}\right\|\left\|x_{m+1}-p^{*}\right\| \\
& +2 \sigma_{m}\left\langle\gamma f\left(p^{*}\right)-\rho W p^{*}, x_{m+1}-p^{*}\right\rangle \\
\leq & 2 \sigma_{m}\left\langle(\rho W-\gamma f) p^{*}, p^{*}-x_{m+1}\right\rangle .
\end{aligned}
$$

Since $\rho \zeta>\gamma \tau$ and $0<\sigma_{m} \leq \frac{1}{\rho\|W\|} \leq \frac{1}{\rho \zeta}$, we get

$$
1-\sigma_{m} \gamma \tau>1-\sigma_{m} \rho \zeta \geq 0
$$


Hence, we can write

$$
\begin{aligned}
\left\|x_{m+1}-p^{*}\right\| \leq & \frac{\left(1-\sigma_{m} \rho \zeta\right)^{2}+\sigma_{m} \gamma \tau}{1-\sigma_{m} \gamma \tau}\left\|x_{m}-p^{*}\right\|^{2} \\
& +\frac{2 \sigma_{m}}{1-\sigma_{m} \gamma \tau}\left\langle(\rho W-\gamma f) p^{*}, p^{*}-x_{m+1}\right\rangle \\
\leq & {\left[1-\frac{2 \sigma_{m}(\rho \zeta-\gamma \tau)}{1-\sigma_{m} \gamma \tau}\right]\left\|x_{m}-p^{*}\right\|^{2}+\frac{\sigma_{m}^{2} \rho^{2} \zeta^{2}}{1-\sigma_{m} \gamma \tau}\left\|x_{m}-p^{*}\right\| } \\
& +\frac{2 \sigma_{m}}{1-\sigma_{m} \gamma \tau}\left\langle(\rho W-\gamma f) p^{*}, p^{*}-x_{m+1}\right\rangle \\
\leq & {\left[1-\frac{2 \sigma_{m}(\rho \zeta-\gamma \tau)}{1-\sigma_{m} \gamma \tau}\right]\left\|x_{m}-p^{*}\right\|^{2} } \\
& +\frac{2 \sigma_{m}(\rho \zeta-\gamma \tau)}{1-\sigma_{m} \gamma \tau}\left[\frac{\left\langle(\rho W-\gamma f) p^{*}, p^{*}-x_{m+1}\right\rangle}{\rho \zeta-\gamma \tau}+\sigma_{m} L\right],
\end{aligned}
$$

where $L$ is a constant satisfying

$$
L \geq \sup _{m \geq 0}\left\{\frac{\rho^{2} \zeta^{2}}{2}\left\|x_{m}-p^{*}\right\|^{2}\right\}
$$

Now, using the condition (B3) and (32), we obtain

$$
\sum_{m=0}^{\infty} \frac{2 \sigma_{m}(\rho \zeta-\gamma \tau)}{1-\sigma_{m} \gamma \tau}>\sum_{m=0}^{\infty} 2(\rho \zeta-\gamma \tau) \sigma_{m}=\infty
$$

and

$$
\limsup _{m \rightarrow \infty}\left(\frac{\left\langle(\rho W-\gamma f) p^{*}, p^{*}-x_{m+1}\right\rangle}{\rho \zeta-\gamma \tau}+\sigma_{m} L\right) \leq 0 .
$$

Therefore, according to Lemma 9, $\left\|x_{m}-p^{*}\right\| \rightarrow 0$ as $m \rightarrow \infty$.

To conclude the proof, note that the variational inequality 18 can be rewritten as

$$
\left\langle(I-\rho W+\gamma f) p^{*}-p^{*}, x-p^{*}\right\rangle \leq 0, \quad x \in \mathcal{F},
$$

which, due to 14 , is equivalent to the fixed point equation

$$
P_{\mathcal{F}}(I-\rho W+\gamma f) p^{*}=p^{*} .
$$

\section{Numerical Example}

Set $\mathcal{H}_{1}=\mathcal{H}_{2}=\mathbb{R}$. Let $C=[0,+\infty)$ and $Q=(-\infty, 0]$. Suppose $A: \mathbb{R} \rightarrow \mathbb{R}$, $W: \mathbb{R} \rightarrow \mathbb{R}, U: C \rightarrow C, V: Q \rightarrow Q$ and $f: \mathbb{R} \rightarrow \mathbb{R}$ are defined by 
Table 1: Results for different initial values

\begin{tabular}{cccc}
\hline \hline No. of iterations & $x_{0}=-1.5$ & $x_{0}=1.0$ & $x_{0}=2.0$ \\
\hline 1 & -1.500000 & 1.000000 & 2.000000 \\
2 & -0.254372 & 0.170616 & 0.336936 \\
3 & -0.049675 & 0.033328 & 0.065775 \\
4 & -0.010527 & 0.007063 & 0.013939 \\
5 & -0.002359 & 0.001583 & 0.003124 \\
6 & -0.000551 & 0.000370 & 0.000730 \\
7 & -0.000133 & 0.000089 & 0.000176 \\
8 & -0.000033 & 0.000022 & 0.000043 \\
9 & -0.000008 & 0.000006 & 0.000011 \\
10 & -0.000002 & 0.000001 & 0.000003 \\
11 & -0.000001 & 0.000000 & 0.000001 \\
12 & -0.000000 & 0.000000 & 0.000000 \\
\hline
\end{tabular}

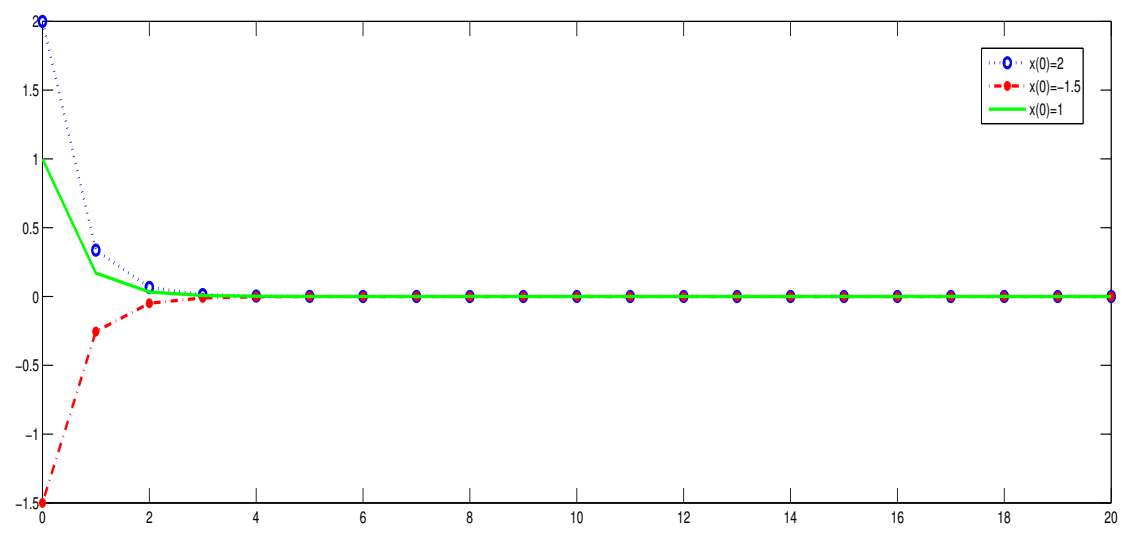

Figure 1: Convergence of $\left\{x_{m}\right\}$ with different initial values for $n=20$.

$A(x)=-x, W(x)=2 x, U(x)=x / 2, V(x)=\sin (x)$, and $f(x)=x / 3, x \in \mathbb{R}$, respectively. Here $A$ is a bounded linear operator, $W$ is a strongly positive bounded linear operator with coefficient $\zeta=2$, and $f$ is a $\tau$-Lipschitzian mapping with coefficient $\tau=1 / 3$. Also, both $U$ and $V$ are non-expansive mappings.

Let $E_{1}: \mathbb{R} \rightarrow \mathbb{R}$ be defined as $E_{1}(x)=2 x$ and $E_{2}: \mathbb{R} \rightarrow \mathbb{R}$ be defined as $E_{2}(x)=-4 x / 5$. For $\lambda=1 / 4$, we compute the resolvent of $E_{1}$ and $E_{2}$ as

$$
J_{\lambda, E_{1}}(x)=\frac{2}{3} x, \quad \text { and } \quad J_{\lambda, E_{2}}(x)=\frac{5}{4} x .
$$

It can be easily seen that $\Omega=\{0\}$ here.

Also, define $G_{1}(z, y)=3 y^{2}+2 z y-5 z^{2}$ and $G_{2}(z, y)=y^{2}-z^{2}$, and put $\phi_{1}(z, y)=\phi_{2}(z, y)=0$. It is easy to see that $G_{1}$ and $G_{2}$ satisfy conditions 
(i)-(iv) of Assumptions 1. Therefore, for $r_{m}=r>0, T_{r}^{G_{1}}(x)$ is non-empty and single-valued for each $x \in C$. Hence, for $r>0$, there exists $z \in C$ such that

$$
G_{1}(z, y)+\frac{1}{r}\langle y-z, z-x\rangle \geq 0 \quad \text { for any } y \in C,
$$

which is equivalent to

$$
3 r y^{2}+(z-x+2 r z) y+\left(x z-5 r z^{2}-z^{2}\right) \geq 0, \quad y \in C .
$$

After solving the above inequality, we get $z=x /(1+8 r)$ for each $r>0$, i.e., $T_{r}^{G_{1}}(x)=x /(1+8 r)$ for each $r>0$. Similarly, $T_{r}^{G_{2}}(x)=x /(1+2 r)$. It can be easily seen that $\mathcal{S}=\{0\}$ here. This implies that $\mathcal{F}=\Omega \cap \mathcal{S}=\{0\}$. Now, let us put $r=1 / 8, \gamma=1$ and

$$
\sigma_{m}=\frac{1}{m+6}, \quad b_{m}=\frac{m+1}{6(m+3)}, \quad \eta_{m}^{1}=\frac{m+3}{m+4}, \quad \xi_{m}^{1}=\frac{m+4}{m+5}
$$

for each $m \geq 1$. Then $\rho \zeta>\gamma \tau$ and the sequences $\left\{\sigma_{m}\right\},\left\{b_{m}\right\},\left\{\eta_{m}^{1}\right\}$, and $\left\{\xi_{m}^{1}\right\}$ satisfy the conditions of Theorems 1,2 and 3 .

In Table 1, we present iterations of the Algorithm for different initial values, which are illustrated on Figure 1. It can be seen, that the constructed sequence $\left\{x_{m}\right\}$ converges to 0 .

Acknowledgments. The authors are very grateful to the anonymous referee for his valuable comments toward the improvement of the paper.

\section{References}

[1] M. Abbas, Y. Ibrahim, A.R. Khan, and M. De la Sen, Split variational inclusion problem and fixed point problem for a class of multivalued mappings in CAT(0) spaces. Mathematics, 7 (2019), no. 8, article number 749. https://doi.org/10.3390/math7080749

[2] M. Abbas, Y. Ibrahim, A.R. Khan, and M. De la Sen, Strong convergence of a system of generalized mixed equilibrium problem, split variational inclusion problem and fixed point problem in Banach spaces. Symmetry, 11 (2019), no. 5, article number 722. https://doi.org/10.3390/sym11050722

[3] Q.H. Ansari and A. Rehan, An iterative method for split hierarchical monotone variational inclusions. Fixed Point Theory Appl., 2015 (2015), article number 121. https://doi.org/10.1186/s13663-015-0368-4

[4] H. H. Bauschke and J. M Borwein, On projection algorithms for solving convex feasibility problems. SIAM review, 38 (1996), no. 3, pp. 367-426. https://doi.org/10.1137/s0036144593251710 
[5] C. Byrne, Iterative oblique projection onto convex sets and the split feasibility problem. Inverse Problems, 18 (2002), no. 2, pp. 441-453. https://doi.org/10.1088/0266-5611/18/2/310

[6] C. Byrne, A unified treatment of some iterative algorithms in signal processing and image reconstruction. Inverse Problems, 20 (2004), no. 1, pp. 103-120. https://doi.org/10.1088/0266-5611/20/1/006

[7] Y. Censor, T. Bortfeld, B. Martin, and A. Trofimov, A unified approach for inversion problems in intensity-modulated radiation therapy. Phys. Med. Biol., 51 (2006), no. 10, pp. 2353-2365. https://doi.org/10.1088/0031-9155/51/10/001

[8] Y. Censor and T. Elfving, A multiprojection algorithm using Bregman projections in a product space. Numerical Algorithms, 8 (1994), no. 2, pp. 221-239. https://doi.org/10.1007/bf02142692

[9] Y. Censor, T. Elfving, N. Kopf, and T. Bortfeld, The multiple-sets split feasibility problem and its applications for inverse problems. Inverse problems, 21 (2005), no. 6, pp. 2071-2084. https://doi.org/10.1088/0266$5611 / 21 / 6 / 017$

[10] Y. Censor, A. Gibali, and S. Reich. Algorithms for the split variational inequality problem. Numerical Algorithms, 59 (2012), no. 2, pp. 301-323. https://doi.org/10.1007/s11075-011-9490-5

[11] H. Chadli and O. Mahdioui, On a system of generalized mixed equilibrium problems involving variational like inequalities in Banach spaces: existence and algorithmic aspects. Adv. Oper. Res., 2021 (2012), Article ID 843486. https://doi.org/10.1155/2012/843486

[12] C. Combettes and P.L. Byrne, Solving monotone inclusions via compositions of non-expansive averaged operators. Optimizations, 53 (2004), no. 5-6, pp. 475-504. https://doi.org/10.1080/02331930412331327157

[13] B. Fan, A hybrid iterative with averaged mappings for hierarchical fixed points and variational inequalities. Numer. Algorithms, 70 (2015), no. 3, pp. 451-467. https://doi.org/10.1007/s11075-014-9956-3

[14] T. Inchan, I. Jitpeera and P. Kumam, A general iterative algorithm combining viscosity method with parallel method for mixed equilibrium problems for a family of strict pseudocontractions. J. Appl. math. Inform., 29 (2011), no. 3-40, pp. 621-639. 
[15] S. Jahedi and M.A. Payvand, System of generalized mixed equilibrium problems, variational inequality, and fixed point problems. Fixed Point Theory Appl. 2016 (2016) article number 93. https://doi.org/10.1186/s13663-016-0583-7

[16] K. Kirk and W.A. Goebel, Topics in metric fixed point theory, Cambridge University Press, Cambridge 28 (1990). https://doi.org/10.1017/CBO9780511526152

[17] P. Majee and C. Nahak, A modified iterative method for capturing a common solution of split generalized equilibrium problem and fixed point problem. RACSAM, 112 (2017), no. 4, pp. 1327-1348. https://doi.org/10.1007/s13398-017-0428-1

[18] P. Majee and C. Nahak, A modified iterative method for split problem of variational inclusion and fixed point problems. J. Comput. and Appl. Math., 37 (2018), no. 4, pp. 4710-4729. https://doi.org/10.1007/s40314018-0596-4

[19] F. Marino, G. Cianciaruso, L. Muglia, Y. Yao, and M.A. Khamsi, A hybrid projection algorithm for finding solutions of mixed equilibrium problem and variational inequality problem. Fixed Point Theory Appl., 2010 (2009), article number 383740. https://doi.org/10.1155/2010/383740

[20] A. Moudafi, A relaxed alternating CQ-algorithm for convex feasibility problems. Nonlinear Anal. Theory Methods Appl., 79 (2013), pp. 117121. https://doi.org/10.1016/j.na.2012.11.013

[21] A. Moudafi. Split monotone variational inclusions, J. Optim. Theory Appl., 150 (2011), no. 2, pp. 275-283. https://doi.org/10.1007/s10957011-9814-6

[22] J.W. Peng, Y.C. Liou and J.-C. Yao, An iterative algorithm combining viscosity method with parallel method for a generalized equilibrium problem and strict pseudocontractions. Fixed Point Theory Appl. 2009 (2009) article number 794178. https://doi.org/10.1155/2009/794178

[23] S. Punpaeng and R. Plubtieng, A general iterative method for equilibrium problems and fixed point problems in Hilbert spaces. J. Math. Anal. Appl. 336 (2007), no. 1, pp. 455-469. https://doi.org/10.1016/j.jmaa.2007.02.044

[24] T. Suzuki, Strong convergence of Krasnoselskii and Mann's type sequences for one parameter non-expansive semigroups without Bochner integrals. J. Math. Anal. Appl., 305 (2005), no. 1, pp. 227-239. https://doi.org/10.1016/j.jmaa.2004.11.017 
[25] W. Takahashi, Split feasibility problem in Banach spaces. J. Nonlinear Convex Anal. 15 (2014), pp. 1349-1355.

[26] S. Takahashi and W. Takahashi, Viscosity approximation methods for equilibrium problems and fixed point problems in Hilbert spaces. J. Math. Anal. Appl., 331 (2007), no. 1, pp. 506-515. https://doi.org/10.1016/j.jmaa.2006.08.036

[27] G. Xu and H.K. Marino, A general iterative method for non-expansive mappings in Hilbert spaces. J. Math. Anal. Appl. 318 (2006), no. 1, pp. $43-52$.

[28] H.K. Xu, An iterative approach to quadratic optimization. J. Optim. Theory Appl., 116 (2003), no. 3, pp. 659-678.

[29] H.K. Xu. A variable Krasnosel'skii-Mann algorithm and the multipleset split feasibility problem. Inverse Problems, 22 (2006), no. 6, pp. 20212034. https://doi.org/10.1088/0266-5611/22/6/007

[30] H.K. Xu, Averaged mappings and the gradient-projection algorithm. J. Optim. Theory Appl., 150 (2011), no. 2, pp. 360-378. https://doi.org/10.1007/s10957-011-9837-z

[31] Q. Yang, The relaxed CQ-algorithm solving the split feasibility problem. Inverse Problems, 20 (2004), no. 4, pp. 1261-1265. https://doi.org/10.1088/0266-5611/20/4/014

[32] Y. Yao, Y.C. Liou and J.C. Yao, Split common fixed point problem for two quasi-pseudo-contractive operators and its algorithm construction. Fixed Point Theory and Appl., 1 (2015), pp. 1-19. https://doi.org/10.1186/s13663-015-0376-4

[33] H. Zhou, Convergence theorems of fixed points for k-strict pseudocontractions in Hilbert spaces. Nonlinear Anal. Theory Methods Appl., 69 (2008), no. 2, pp. 456-462. https://doi.org/10.1016/j.na.2007.05.032

[34] L.J. Zhu, Y.C. Liou, Y. Yao, and C.C. Chyu, Algorithmic and analytical approaches to the split feasibility problems and fixed point problems. Taiwan. J. Math. 17 (2013), no. 5, pp. 1839-1853. https://doi.org/10.11650/tjm.17.2013.3175

Shamshad Husain

Department of Applied Mathematics ,

Faculty of Engineering and Technology,

Aligarh Muslim University, Aligarh-202002, India.

s_husain68@yahoo.com 
Mohd Asad

Department of Applied Mathematics, Faculty of Engineering and Technology, Aligarh Muslim University, Aligarh-202002, India. masad19932015@gmail.com

Mubashshir Uddin Khairoowala

Department of Applied Mathematics , Faculty of Engineering and Technology, Aligarh Muslim University, Aligarh-202002, India. mkhairoowala@gmail.com

Please, cite to this paper as published in Armen. J. Math., V. 13, N. 7(2021), pp. 132 https://doi.org/10.52737/18291163-2021.13.7-1-32 